\title{
Öffentliche Mobilität und neue Mobilitätsdienstleistungen - Rahmenbedingungen und Gestaltungsperspektiven
}

\section{Christina Wolking}

\section{Einleitung}

Der Mobilitätssektor befindet sich in einem umfassenden Transformationsprozess. Gesellschaftliche Makroentwicklungen, wie die Urbanisierung, Individualisierung, demographischer Wandel und Digitalisierung, eröffnen neue Möglichkeiten, führen aber andererseits auch zu erweiterten Anforderungen an das Mobilitätssystem. Die veränderten Rahmenbedingungen auf übergeordneter Ebene stoßen einen Wandel in Hinblick auf das Mobilitätsverhalten, auf das Mobilitätsangebot, aber auch auf die Leitbilder und Strategien der Politik an. Die Debatte um eine Verkehrswende verdeutlicht die Notwendigkeit von Veränderungen des Verkehrssystems. Durch den kontinuierlich hohen Anteil an den Treibhausgasemissionen in Deutschland kommt dem Verkehrssektor, als zweitgrößter Emittent nach der Energiewirtschaft, eine entscheidende Rolle im Kampf gegen den Klimawandel zu. Bis zum Jahr 2050 sollen die Emissionen weitestgehend neutralisiert werden, bis 2030 soll bereits eine Verminderung der Emissionen im Vergleich zu 1990 um 40 bis $42 \%$ erfolgen (BMU 2016, S. 7; Agora Verkehrswende 2017, S. 7).

Die negativen Folgen des hohen Verkehrsaufkommens stehen der großen Bedeutung individueller Mobilität gegenüber. Sie bestimmt über Lebensqualität und den Grad der gesellschaftlichen Teilhabe und muss im Sinne der Daseinsvorsorge vom Staat für alle Menschen gesichert werden (siehe den Beitrag von Schwedes und Ringwald in diesem Band). Um diese beiden Ziele zu vereinen

C. Wolking $(\bowtie)$

Technische Universität Berlin, Berlin, Deutschland

E-Mail: christina.wolking@tu-berlin.de

(C) Der/die Autor(en) 2021 
bedarf es einer Orientierung an dem Ziel, eine bedarfsgerechte Mobilität mit weniger Verkehr zu fördern (Becker 2001, S. 4).

Im Zentrum der Förderung umweltverträglicher und sozial gerechter Mobilität stehen die Verkehrsmittel des Umweltverbundes. Dem öffentlichen Verkehr kommt dabei als Rückgrat des Umweltverbundes eine tragende Funktion in der Gestaltung eines nachhaltigen Verkehrssystems zu. Häufig wird er dem MIV als umweltfreundliche Alternative gegenübergestellt, mit dem Ziel den Verkehr zu dekarbonisieren und die negativen Effekte der autodominierten Mobilitätskultur zu senken (Dziekan und Zistel 2018, S. 361).

Mit dem Leitbild einer Integrierten Verkehrsplanung wird ein ganzheitlicher Planungsansatz verfolgt, welcher die Ziele einer nachhaltigen Verkehrsentwicklung und die Sicherung der sozialen Teilhabe in den Fokus stellt. Gestaltungsdimensionen beziehen sich auf die Bereiche der Infrastruktur, den Verkehrsprozess sowie der Mobilität. Wie bereits einleitend beschrieben, sind Strategien und Maßnahmen unter der Berücksichtigung der normativen Ansprüche unterschiedlicher Akteure in einem kooperativen und transparenten Planungsprozess, unabhängig von politischen, fachlichen und räumlichen Grenzen zu konzeptionieren (Schwedes und Rammert 2020, S. 24; Schwedes et al. 2018, S. 4).

Während die Emotionen von Verkehrsteilnehmenden in der Verkehrsplanung häufig vernachlässigt und Entscheidungen anhand objektiv messbarer Faktoren getroffen werden, wird bei der Integrierten Verkehrsplanung auch der Einfluss subjektiver Empfindungen auf das Mobilitätsverhalten mit einbezogen. Für eine erfolgreiche Umgestaltung des Verkehrssystems ist es essenziell, neben der Orientierung an harten Faktoren auch gesellschaftliche Rahmenbedingungen zu berücksichtigen und Beweggründe für das Mobilitätsverhalten zu verstehen.

Öffentliche Mobilität wird in diesem Sammelband als Verschmelzung, als Annäherung, als Kompromiss der individuellen Automobilität und des öffentlichen Verkehrs diskutiert. Die Frage, was dabei genau Öffentliche Mobilität bedeutet, was sie umfasst, wie sie gestaltet werden kann, welche Herausforderungen sich ergeben und welche Akteure dabei von Bedeutung sind, rücken einmal mehr in den Fokus, da die beschriebenen Veränderungen im Mobilitätssektor ein sogenanntes, window of opportunity " öfnen und die Verkehrswende begünstigen können. Der Ansatz ,Nutzen statt Besitzen“ und die Angebotsperspektive ,Mobilität als Dienstleistung ' wird in Kombination mit geteilter Mobilität vor allem seitens der Anbietenden als Potenzial beschrieben, um die Abhängigkeit vom Besitz eines privaten Pkw aufzulösen. Beispielsweise können für Car-Sharing zwar positive Effekte auf die Reduktion des Pkw-Besitz 
dokumentiert werden, jedoch stehen bei vielen Studien Personen im Fokus, die bereits Kund*innen sind, was die Ergebnisse wiederum verfälschen kann. Im wissenschaftlichen Diskurs wird vor diesem Hintergrund auch die Notwendigkeit betont, die Angebote im Rahmen eines ganzheitlichen Planungsansatzes zu integrieren (Giesel und Nobis 2016, S. 223; Zhou et al. 2020, S. 338).

Die Entstehung ,neuer Mobilitätsdienstleistungen', die sich in einem Bereich zwischen kollektivem Verkehr und Individualverkehr sowie öffentlichem und privatem Verkehr verorten lassen, erfolgt vorwiegend im urbanen Raum. Die überwiegend von der Wirtschaft getriebenen Angebote sind mit neuen Geschäftsmodellen verbunden und werden unter anderem von der Automobil- oder der IT-Branche zur Verfügung gestellt. Je nach Angebotsform werden sie als Chance oder Risiko für die Verkehrswende und die Gestaltung eines nachhaltigen Mobilitätsangebots gesehen (Karlsson et al. 2020, S. 286). Die Angebote befinden sich dabei häufig in einer Testphase und bewegen sich in einem rechtlich neuen Bereich, wodurch sie - unabhängig von ihrem Potenzial - einerseits räumlich, aber andererseits auch zeitlich begrenzt verfügbar sind. Eine integrierte ganzheitliche Planung erweist sich unter den aktuellen Rahmenbedingungen bislang als schwierig. Die Veränderung des Mobilitätsverhaltens und die Integration der Nutzung dieser Mobilitätsdienstleistungen in vorhandene Alltagsroutinen sind nur bei entsprechender Verfügbarkeit und zuverlässigem Zugang möglich. Entscheidend für die Ausgestaltung der zukünftigen Öffentlichen Mobilität ist in diesem Sinne auch die Analyse der Interessen unterschiedlicher Akteure, insbesondere da sich ein komplexes Spannungsfeld abzuzeichnen scheint, das sich zwischen verkehrspolitischen Zielen, wirtschaftlichen Interessen und den individuellen Bedürfnissen der Verkehrsteilnehmenden aufspannt. So stehen Ziele, wie die Reduktion des Straßenverkehrs und die Förderung einer nachhaltigen und bedarfsgerechten Mobilität, unter anderem der Profitorientierung und dem Ziel, erlösstarke Verkehre zu erzielen, gegenüber (Docherty et al. 2018, S. 119). Um eine Orientierung an verkehrs- und stadtplanerischen Zielen zu gewährleisten, ist die Steuerung und Koordination der öffentlichen Mobilitätsangebote durch die Kommunen essenziell (Hausigke und Kruse in diesem Band).

Im weiteren Verlauf werden zunächst Defizite der sich gegenüberstehenden Systeme des öffentlichen Verkehrs und des privaten Verkehrs beleuchtet. Darauf aufbauend werden Rahmenbedingungen und Elemente einer veränderten Mobilität sowie Potenziale und Risiken neuer Mobilitätsdienstleistungen erläutert. Im Sinne einer zukünftigen Öffentlichen Mobilität sollen abschließend Gestaltungsperspektiven für neue Mobilitätsdienstleistungen aufgezeigt werden. 


\section{$1 \quad$ Der Reformbedarf des öffentlichen Verkehrs}

Die Gewährleistung der Daseinsvorsorge wird aktuell entsprechend des Regionalisierungsgesetzes (RegG) weitestgehend durch den öffentlichen Personennahverkehr übernommen, dessen Ziel es ist, eine Grundversorgung an Mobilität mit marktfähigen Preisen zu bieten (Institut für Mobilitätsforschung 2006, S. 40). Dabei konkretisiert sich dessen Ziel damit, eine ,ausreichende Bedienung der Bevölkerung mit Verkehrsleistungen“ (RegG§1(1)) sicherzustellen. Zudem wird der ÖPNV als die ,allgemein zugängliche Beförderung von Personen mit Verkehrsmitteln im Linienverkehr [bezeichnet], die überwiegend dazu bestimmt ist, die Verkehrsnachfrage im Stadt-, Vorort- oder Regionalverkehr zu befriedigen“ (RegG §2). Der ÖV steht als Kollektivverkehr für die örtliche und zeitliche Bündelung von Fahrten und ist zudem fahrplan- und stationsgebunden (Dziekan und Zistel 2018, S. 348). Gegen eine Gebühr, die u. a. nach Entfernung, Zeit oder Art des Service berechnet wird, erhalten Nutzende Zugang zu der Dienstleistung. Bereitgestellt wird der ÖV von den auf Landesebene definierten Aufgabenträgern (Kommunen und Landkreise), die wiederum in den Nahverkehrsplänen - orientiert an PBefG und den ÖPNV-Gesetzen der Länder - die Ausgestaltung des ÖPNV spezifizieren (Karl 2014, S. 89, 94; Harman et al. 2012, S. 287).

Auf europäischer Ebene werden in der DIN EN 13816 Kategorien definiert, anhand welcher die Qualität des ÖPNV beurteilt werden kann. Dementsprechend ist die Qualität bezogen auf die Erschließung, Bedienung, Verbindung, Ausrüstung, Service und Umwelt zu messen. Die Norm definiert dabei keine Qualitätsniveaus, sondern enthält die Aufforderung an Auftraggebende und -nehmende in den jeweiligen Bereichen eigene Standards zu konkretisieren. Es wird zudem darauf hingewiesen, dass nicht ausreichend Mittel zur Verfügung stehen, um allein durch Pull-Maßnahmen und eine gesteigerte Attraktivität des ÖPNV Verhaltensänderungen in der Verkehrsmittelwahl zu erwirken (König 2007).

Das Konzept der Daseinsvorsorge und das Selbstverständnis des öffentlichen Verkehrs stehen angesichts aktueller Entwicklungen zunehmend auf gesellschaftlicher, politischer und wissenschaftlicher Ebene in der Kritik. Das bemängelte Minimalprinzip des ÖV drückt sich in den verwendeten Begrifflichkeiten der ,Grundversorgung', der , ausreichenden Bedienung' sowie dem Anspruch , die Verkehrsnachfrage zu befriedigen' aus. Die dem ÖV zugeschriebene hohe Bedeutung als attraktive und umweltfreundliche Alternative zum MIV spiegelt sich nicht in den Ausgestaltungsmöglichkeiten wider. Insbesondere wird die Auffassung der Kund*innen als ,Beförderungsfälle“ kritisiert, in deren Bezeichnung 
sich die vielfach thematisierte mangelnde Nutzer*innenorientierung reflektiert. Dem Bedarf nach individueller Mobilität kann der traditionelle öffentliche Verkehr nach dieser Auffassung bereits per Definition nicht gerecht werden (Karl 2008, S. 12; Schwedes 2014a, S. 17).

Die Gestaltung eines ÖV-Systems, das den Anforderungen und Bedürfnissen der Nutzer*innen gerecht wird und einen sozial gerechten Zugang garantiert, stellt für die Anbietenden eine große Herausforderung dar. Die Planung erfolgt viele Jahre im Voraus und basiert auf Verkehrsprognosen, sodass reaktives Handeln und sofortige Anpassungen nur eingeschränkt möglich sind. Während die Fahrgastzahlen des ÖV in Städten steigen und Kapazitäten knapp sind, nehmen die Kosten für ein leistungsstarkes ÖV-System mit abnehmender Siedlungsdichte zu, sodass die Wirtschaftlichkeit der liniengebundenen Bedienung in Randgebieten häufig infrage gestellt wird (Deffner et al. 2014, S. 226). Zweifel werden zudem daran geäußert, ob der ÖPNV den anvisierten potenziellen Anstieg der Nutzer*innenzahlen aufgrund mangelnder Kapazitäten und seiner geringen Anpassungsfähigkeit bewältigen könne. Als Erklärungsansatz für die geringe Flexibilität wird eine frühzeitige staatliche Regulierung genannt, die Beschränkungen in der Ausgestaltung und der Diversifizierung der Bedienungsformen nach sich zog und einen Fokus auf den im Vergleich zum Busverkehr weniger flexiblen Schienenverkehr legte. Der Gestaltungsspielraum für Produktinnovationen und neue Bedienkonzepte ist gering, da das ÖV-System überwiegend an veraltete Regulierungen und Gesetze gebunden ist. Entsprechend entsteht die Notwendigkeit, bestehende Gesetze zu überprüfen und so zu erweitern, dass sie den veränderten Rahmenbedingungen gerecht werden (z. B. Car-Sharing-Gesetz oder Mobilitätsgesetz in Berlin) (Karl 2008,S. 23 f.; Karlsson et al. 2020, S. 292).

Aus Sicht der (potenziellen) Nutzer*innen werden in Abhängigkeit des jeweiligen Bezugsraums unterschiedliche Aspekte bemängelt und führen zu einer verminderten Attraktivität. Während in städtischen Randgebieten starre Linienführungen, zeitliche Gebundenheit an Fahrpläne und geringe Taktfrequenzen in den Nebenverkehrszeiten die Flexibilität einschränken, wird in Stadtzentren und zu Hauptverkehrszeiten die hohe Auslastung sowie die Bündelung von Fahrten im Kollektivverkehr zur Belastung der Nutzer*innen. Aus Nutzer*innensicht widerspricht diese Form der Fortbewegung dem Bedürfnis nach Autonomie und Selbstbestimmtheit, sodass die geringe Privatsphäre bei voller Auslastung von Bus und Bahn als unangenehm eingestuft wird (Meyer 2014, S. 172). Weitere Hindernisse für die Nutzung des öffentlichen Verkehrsangebots sind der $\mathrm{Zu}$ - und Abgang zur Haltestelle (die sogenannte erste und letzte Meile im ÖV), (subjektiv empfundene) Zeitnachteile, die Notwendigkeit von häufigem Umsteigen und 
Informationsdefizite bezüglich Verfügbarkeit und Zugang. Verstärkt werden diese Beweggründe bei der direkten Verfügbarkeit des eigenen Pkw (Karl 2008, S. 22). Hervorzuheben ist, dass Nutzer*innen Aspekte in den zuvor beschriebenen Qualitätskategorien bezüglich Erschließung, Bedienung, Verbindung, Ausrüstung sowie Service bemängeln.

Angelehnt an die aufgeführten Defizite im ÖV wird die Frage thematisiert, inwiefern der ÖV der Rolle als Hoffnungsträger auf dem Weg zu einer nachhaltigen Mobilität gerecht werden kann und ob er als Rückgrat des Umweltverbundes die Basis für eine realistische Alternative zum MIV darstellt. Denn erst durch innovative und konkurrenzfähige Angebote zum MIV, die auch aus Sicht der Nutzer*innen attraktiv sind, kann der Verkehr nachhaltig gestaltet werden und eine ,Entkopplung der Zwangsautomobilität" stattfinden (Deffner et al. 2014, S. 204).

\section{Die Dominanz der Automobilität}

Die Gründe für die nach wie vor hohe Dominanz des Pkw sind vielschichtig. Zu differenzieren ist prinzipiell zwischen Regionen, wo es keine oder kaum Alternativen zum privaten Pkw gibt und jenen, in denen trotz objektiv betrachteter vielfältiger Ortsveränderungsmöglichkeiten der MIV eine entscheidende Rolle spielt. Neben den individuellen Rahmenbedingungen und persönlichen Präferenzen wird die Mobilität auch von übergeordneten Rahmenbedingungen, wie unter anderem der räumlichen Struktur, der Gesellschaft und der Kultur beeinflusst. Vergangene gesellschaftliche Entwicklungen und politische Entscheidungen führten zu einer sogenannten Pfadabhängigkeit, sodass die deutsche ,Autokultur' und die hohe Affinität zum privaten Pkw auch historisch gewachsen sind (Canzler 2012, S. 317). Mit der Massenmotorisierung in den 1950er Jahren, durch die der Erwerb eines eigenen $\mathrm{Pkw}$ für einen Großteil der Bevölkerung bezahlbar wurde, rückte der öffentliche Verkehr in den Hintergrund, da mit dem motorisierten Individualverkehr (MIV) der Mobilitätsbedarf unabhängig von den staatlichen Dienstleistungen abgedeckt werden konnte. Der private Pkw schien die optimale Lösung zu sein, für eine schnelle, flexible und komfortable Möglichkeit, von Tür zu Tür zu kommen und hat so die Mobilität, den Verkehr, aber auch den Raum und die Umwelt seit seiner Existenz grundlegend verändert (Rammler 2014, S. 41; Pavone 2015, S. 400).

Mit den neu gewonnen und flexiblen Bewegungsmöglichkeiten durch das Auto, stellten große Distanzen in der Überwindung kein Problem mehr dar, sodass neue Wohnräume erschlossen wurden. Im Zuge dessen folgten Suburbanisierungsprozesse, Zersiedelung und die Ausdehnung von Wohngebieten. Eine Alternative 
zum MIV gab es in diesen Regionen selten, war schwer umzusetzen oder wurde bei der Planung nicht mitgedacht, sodass in der Folge die Abhängigkeit vom Pkw zusätzlich stieg. Weitere Ursachen und Entwicklungen, die bis heute die , deutsche Autokultur' bedingen sind - um nur einige Beispiele $\mathrm{zu}$ nennen - die steigende Arbeitsteilung, die enge Verbindung zwischen der Automobilwirtschaft und der deutschen Politik sowie das Paradigma des Automobils als deutsches Statussymbol (Kutter 2016, S. 212; Schade und Schlag 2007, S. 27; Menzl 2013, S. 62).

Mit der Ausdehnung der Siedlungsflächen nahm auch die Anzahl und die Länge der notwendigen Wege zu, da Arbeiten, Wohnen, sich versorgen und Freizeitaktivitäten nicht am gleichen Ort möglich waren. Verkehr gilt bis heute entsprechend als Grundvoraussetzung, um bestimmte Bedürfnisse zu erfüllen, sodass die Verkehrserreichbarkeit nicht kurz- oder mittelfristig vernachlässigt werden kann und Alternativen gefördert und etabliert werden müssen. Das Mobilitätsangebot variiert in Abhängigkeit des jeweiligen Raums, der Siedlungsstruktur, den physischen Gegebenheiten, den ökonomischen sowie gesellschaftlichen Rahmenbedingungen (Kutter 2016, S. 212).

Sofern Alternativen zum privaten Pkw vorhanden sind, spielen neben der Verfügbarkeit der Verkehrsmittel auf individueller Ebene auch das Empfinden und die subjektive Wahrnehmung der Mobilitätsangebote eine Rolle. Demnach kann das Mobilitätsverhalten und schließlich auch die Verkehrsmittelwahl aufgrund persönlicher Einstellungen, Leitbilder und Normen sowie Fähigkeiten und Gewohnheiten trotz identischer objektiver Voraussetzungen unterschiedlich ausfallen (Schwedes et al. 2018, S. 5; Gertz 2013, S. 42). Faktoren wie Zeit, Unabhängigkeit, Komfort und das Motiv des Weges werden als jene mit dem größten Einfluss auf das Mobilitätsverhalten identifiziert (Rölle 2005, S. 52; Kagermeier 2011, S. 737).

Die komplexen Aktivitätsräume der heutigen Gesellschaft erfordern ein hohes $\mathrm{Maß}$ an Flexibilität. Bei der häufigen Gegenüberstellung von Individual- und Kollektivverkehr wird der Individualverkehr unter anderem aufgrund zeitlicher und räumlicher Unabhängigkeit bevorzugt (Schneider 2018, S. 13; Fraedrich und Lenz 2015, S. 690). Bei Verfügbarkeit eines Pkw werden die alternativen Verkehrsmittel zumeist verdrängt und häufiger Wege als ursprünglich geplant und notwendig mit dem Pkw absolviert, wie Canzler (2016, S. 111) mit dem Mechanismus des, mental car' beschreibt. Im Moment der Nutzung stehen die klaren Vorteile der eigenen Pkw-Nutzung im Vordergrund, die entstehenden Beeinträchtigungen auf gesellschaftlicher Ebene und die negativen Effekte werden externalisiert, wenn auch nicht bewusst. Auch wenn Alternativen zum Pkw vorhanden sind, stellen Verhaltensänderungen eine große Herausforderung für die Planung dar, da in den überwiegenden Fällen eine Lücke bzw. ein Widerspruch 
zwischen dem Wissen und der tatsächlichen Handlung besteht. In diesem Kontext wird auch von Strategien zur Reduktion der kognitiven Dissonanz gesprochen (Canzler 2016, S. 111; Schade und Schlag 2007, S. 28 f.).

Die zuvor beschriebenen Aspekte beschreiben ein Dilemma, mit dem die Verkehrsplanung sich konfrontiert sieht. Die Vor- und Nachteile der sich gegenüberstehenden Verkehrssysteme des öffentlichen Verkehrs und des Motorisierten Individualverkehrs stehen sich auf unterschiedlichen Ebenen diametral gegenüber, wodurch hier eine Art Konkurrenz zwischen den gemeinwohlorientierten Interessen einerseits und den individuellen Interessen andererseits entsteht. Basierend auf den zuvor beschriebenen Defiziten des ÖV, dem wachsenden individuellen Mobilitätsbedarf der Menschen sowie den negativen Auswirkungen des dominierenden MIV begründet sich nicht nur die vielfach geforderte Reform des ÖV, sondern vielmehr die Notwendigkeit, das Verkehrssystem als Ganzes zu überdenken und die Vorteile der beiden Systeme zu integrieren (Schwedes 2014a, S. 21). Mit dem Leitbild der Integrierten Verkehrsplanung sowie dem Ansatz, multimodales Mobilitätsverhalten zu fördern, steht eine Integration der verschiedenen Verkehrsträger und eine flexible Nutzung bereits im Fokus. Deutlich wird zudem, dass den Bedarfen der Nutzer*innen eine entscheidende Rolle in der Gestaltung zukommen muss; einerseits bezüglich einer Orientierung an den spezifischen Anforderungen der Menschen, andererseits durch die Beeinflussung des Mobilitätsverhaltens im Sinne des strategischen Mobilitätsmanagements.

Das Verständnis und die Wahrnehmung, dass der ÖV dabei ausschließlich für kollektiven Verkehr steht und der Motorisierte Individualverkehr die individuelle flexible Mobilität liefert, muss in diesem Sinne aufgelöst werden. Es gilt, eine Transformation von konkurrierenden Systemen zu kooperierenden Systemen zu gestalten. Dabei sind Staat und Wirtschaft zentrale Akteure und verkörpern unterschiedliche Interessen. Entscheidend für die zukünftigen Entwicklungen ist die Schaffung von förderlichen Rahmenbedingungen auf unterschiedlichen Ebenen und die notwendige Steuerung seitens der Politik. So gilt es, den öffentlichen Verkehr im Sinne einer Öffentlichen Mobilität weiter zu denken. Essentiell ist die Sicherung der gesellschaftlichen Teilhabe für alle unter Berücksichtigung sozialer, ökologischer und ökonomischer Aspekte einer nachhaltigen Verkehrsentwicklung.

\section{3 Öffentliche Mobilität}

Neue Mobilität, zukunftsorientierte Mobilität, innovative oder vernetzte Mobilität, - die Liste der Begrifflichkeiten, die mit Transformationen im Verkehrssektor einhergehen und Anspruch erheben, einen Lösungsansatz für die zuvor 
beschriebenen Defizite zu liefern, ist lang. Auch der Terminus ,öffentliche Mobilität" wird immer häufiger genannt. Dennoch hat sich der Gebrauch im wissenschaftlichen und gesellschaftlichen Diskurs noch nicht etabliert. Bislang mangelt es an einer eindeutigen Definition von Öffentlicher Mobilität. Oftmals wird, genau wie bei der Differenzierung zwischen Verkehr und Mobilität, keine klare und bewusste Grenze gezogen und durch weitere Adjektive im Titel wie z. B. „,bedarfsorientierte öffentliche Mobilität“, ,individuelle öffentliche Mobilität“ oder ,zukunftsfähige öffentliche Mobilität“ beschrieben, was unter öffentlicher Mobilität zu verstehen ist oder was gar das normative Ziel dahinter ist (z. B. VDV 2019; ADAC e. V. 2020; Trapeze Switzerland GmbH 2020).

\subsection{Zwischen Verkehr und Mobilität.}

Um Öffentliche Mobilität besser vom öffentlichen Verkehr abgrenzen zu können und als Grundlage für die Unterscheidung zwischen Mobilitätsdienstleistungen und Verkehrsdienstleistungen ist es erforderlich, einen Blick auf die Definitionen von Mobilität und Verkehr zu werfen und das hier zugrunde liegende Verständnis zu erläutern. Dies geschieht vor allem angesichts dessen, dass in Wissenschaft und Politik auf unterschiedliche Definitionen zurückgegriffen wird und in der Verwendung oftmals keine klare Differenzierung stattfindet (Schwedes et al. 2018, S. 5). Verkehr wird als die ,realisierte Ortsveränderung von Personen, Gütern und Nachrichten" definiert und kann in diesem Sinne als Prozess und physische Bewegung verstanden werden (Nuhn und Hesse 2006, S. 18). Darüber hinaus wird Verkehr im Kontext der Integrierten Verkehrsplanung auch als Gesamtsystem verstanden, das sich aus der Infrastruktur, dem Verkehrsprozess sowie dem Mobilitätsverhalten der Menschen ergibt (Schwedes et al. 2018, S. 6). Die Begriffsdefinitionen von Mobilität variieren zwischen unterschiedlichen Wissenschaftsdisziplinen. Auch innerhalb der Verkehrs- und Mobilitätsforschung stehen sich verschiedene Definitionsansätze gegenüber, die unter anderem „Mobilität als Bewegung“ oder „Mobilität als Beweglichkeit“ betrachten (Gerike 2006, S. 22). In diesem Beitrag wird Bezug zur Definition von Schwedes et al. (2018) genommen, die Mobilität definieren als ,die subjektive Ausprägung der Ortsveränderungsmöglichkeiten [, welche aus] den räumlichen, physischen, ökonomischen und gesellschaftlichen Rahmenbedingungen und deren subjektiver Wahrnehmung “ (ebd., S. 5) resultiert. Diese Definition betont, dass das objektiv vorhandene Verkehrsangebot durch einen subjektiven Filter wahrgenommen wird. Einen Einfluss haben also einerseits die Qualität und die Ausgestaltung des Angebots, andererseits die persönlichen Einstellungen, Fähigkeiten und 
Werte der Individuen. Neben den beständigen Rahmenbedingungen haben auch weitere variierende Aspekte, wie das Motiv der Fortbewegung, das Ziel der Ortsveränderung und deren Zeitpunkt, erheblichen Einfluss auf die Verkehrsmittelwahl. Das Mobilitätsverhalten ergibt sich demnach aus einem komplexen System korrelierender Einflussfaktoren subjektiver und objektiver Natur (siehe den Beitrag von Rammert in diesem Band).

Während sich Verkehr auf das Gesamtsystem bezieht und eine angebotsorientierte Perspektive impliziert (Infrastruktur und Fortbewegungsmittel stehen im Fokus), bezieht sich Mobilität auf das Individuum und die Bedürfnisse. Die Begrifflichkeiten unterscheiden sich auch bezüglich der Emotionen, die sie hervorrufen. Lange galt Verkehr als positives Zeichen, das auf Wohlstand und Wachstum deutete, doch angesichts der veränderten Rahmenbedingungen, wie dem Klimawandel und einem gesteigerten Umweltbewusstsein, wird mittlerweile von einem Paradigmenwechsel gesprochen. Verkehr wird mit seinen negativen Auswirkungen in Verbindung gebracht, während Mobilität als die Möglichkeit zur Bewegung positiv besetzt ist (Gegner und Schwedes 2014, S. 67). Auch in Bezug auf öffentlichen Verkehr lässt sich eine negative Konnotation verzeichnen, die nicht zuletzt auf das Image und die zuvor bereits beschriebenen Defizite aus Nutzer*innensicht zurückzuführen sind. Der ÖV wird mit Fremdbestimmung verbunden, die Nutzer*innen als sogenannte ,Captives' bezeichnet. Gleichzeitig wird mit der ,Automobilität ' Autonomie und Flexibilität impliziert (Gegner und Schwedes 2014, S. 67). Bereits bei der Betrachtung der Definitionen von Mobilität und Verkehr wird, ergänzend zu der viel diskutierten Reform des ÖPNV, auch die Notwendigkeit eines neuen begrifflichen Verständnisses erkennbar.

\subsection{Die Bedeutung von öffentlich und privat}

Vor dem Hintergrund, dass mit dem Ziel öffentliche Mobilität zu gewährleisten und der Erweiterung des Verkehrsangebots eine Annäherung des öffentlichen Verkehrs und des Individualverkehrs einhergeht, folgt eine Auseinandersetzung mit den Bedeutungen von ,öffentlich' und ,privat'.

Öffentlich bedeutet per Definition ,allgemein zugänglich“. Diesem Verständnis liegt auch das ,öffentlich` im öffentlichen Verkehr zugrunde (Dudenredaktion 2020). Die allgemeine Zugänglichkeit kann dabei auf zwei Ebenen bezogen werden: zum einen auf die räumliche Ebene, die Verfügbarkeit indiziert und zum anderen auf die soziale Ebene, welche sich auf die Nutzbarkeit bezieht (Hoffjann und Arlt 2015, S. 7 f.). Im weiteren Sinne bedeutet dies, dass Verkehrsangebote und Infrastruktur, die durch die öffentliche Hand bereitgestellt werden, 
zugänglich, verfügbar und für die Allgemeinheit - also für Personen mit unterschiedlichen Voraussetzungen - nutzbar sein müssen. Die Nutzbarkeit und die Verfügbarkeit implizieren darüber hinaus das Potenzial/die Option/die Möglichkeit, die hinter ,öffentlich“ steht. Das heißt, dass öffentlicher Zugang inklusiv sein muss und mit Wahlfreiheit einhergeht. $\mathrm{Zu}$ differenzieren ist auch zwischen frei und gratis, was nicht gleichzusetzen ist. Etwas kann zugleich öffentlich sein und dennoch Begrenzungen durch beispielsweise Bezahlmechanismen vorweisen. Ein Kostenausgleich erfolgt bei öffentlicher Nutzung dabei je Einheit (z. B. Anzahl, Zeit, Distanz).

Dem „Öffentlichen“ kann das „Private“/das „Exklusive“ gegenübergestellt werden (Habermas 1991, S. 55). Im Gegensatz zum allgemein Zugänglichen beschränkt sich das Private auf den Zugang einer spezifischen Person oder einer Personengruppe. Mittels einer rechtlichen Grenze und/oder einer physischen Barriere wird die Zugänglichkeit für weitere Personen ausgeschlossen. Hervorzuheben ist vor diesem Hintergrund nochmals der Gestaltungsanspruch Öffentlicher Mobilität, die gesellschaftliche Teilhabe in Form von Mobilität für alle ermöglichen möchte. Dementsprechend wird der barrierefreien Gestaltung eine besondere Rolle zuteil. Zuvor wurden bereits die räumliche sowie die soziale Ebene als Bezugsdimensionen des Öffentlichen genannt. Darüber hinaus kann eine materielle Dimension ergänzt werden, die sich auf Gegenstände/Objekte bezieht sowie eine mediale Dimension, die Informationen und Nachrichten betrifft.

Geht es um Objekte - in diesem Kontext um Beförderungsmittel - wird im Kontext des Privaten zwischen Besitz und Eigentum unterschieden. Privateigentum umschreibt dabei, laut BGB § 903 die vollumfängliche Sachherrschaft über ein Gut, sodass der/die Eigentümer*in mit der Sache nach Belieben verfahren kann. Besitz impliziert laut BGB $\S 854$ die temporäre Herrschaft über eine Sache, wodurch auch Mietgegenstände oder geliehene Objekte als Besitz bezeichnet werden. Besitz und Eigentum umschreiben das Verhältnis zwischen Gut und Konsument*in, weshalb die Nutzung von Fahrzeugen an sich den Besitz nicht ausschließt. Dementsprechend trifft die Bezeichnung ,Nutzen statt besitzen im Kontext der Sharing-Economy nicht eindeutig den Sachverhalt und wird als semantisch unscharf kritisiert. So argumentieren Schlag und Rößger (2019, S. 11), dass folgende Formulierung eine adäquate Alternative sei: „temporär besitzen und geteilt nutzen statt dauerhaft exklusiv aneignen“".

Da öffentlich ,allgemein zugänglich ' bedeutet, wird diesbezüglich primär eine geteilte oder eine gemeinschaftliche Nutzung verbunden. Im Zusammenhang mit dem traditionellen öffentlichen Verkehr geht damit eine kollektive Nutzung einher. Um dem Anspruch des, Öffentlichen` gerecht zu werden, muss 
die gemeinschaftliche Nutzung jedoch nicht zwingenderweise zeitgleich, also kollektiv erfolgen. Die geteilte Nutzung kann ebenso durch den temporären Zugang von Besitz gestaltet werden. Die sogenannte, eigentumslose Nutzung', die temporären Besitz beschreibt, fällt rein rechtlich allerdings in den Bereich des Privaten (z. B. bei Sharing-Angeboten). Bei der Betrachtung von Akteuren ist zudem zu berücksichtigen, dass auch hier die öffentliche der privaten Sphäre gegenübersteht. Öffentliche Akteure umfassen dabei die öffentliche Hand, die durch Repräsentant*innen im öffentlichen Dienst aus Politik und Verwaltung vertreten wird. Die privaten Akteure werden durch wirtschaftliche sowie zivilgesellschaftliche Institutionen verkörpert. Dies verdeutlicht, dass in der Praxis Grenzen zwischen privater und öffentlicher Sphäre mit der zunehmenden Flexibilisierung und Diversifizierung der Verkehrsangebote und der Anbietenden verschwimmen und sich eine klare Abgrenzung zwischen privat und öffentlich als schwierig gestaltet. So kann ein Angebot zwar allgemein zugänglich sein, aber dennoch mit privater/individueller Nutzung verbunden sein. Ein allgemein zugängliches und öffentliches Verkehrsangebot schließt zudem nicht aus, dass dieses durch private Anbieter bereitgestellt wird.

Vor diesem Hintergrund kann erneut auf die Notwendigkeit verwiesen werden, einer klaren normativen Vorstellung Öffentlicher Mobilität zu folgen und Anforderungen an die Ausgestaltung der neuen Mobilitätsdienstleistungen zu stellen.

Aus der Perspektive der Anbietenden lässt sich das Teilen anhand zwei unterschiedlicher Motivationen erklären. Einerseits kann Teilen eine sozial normative Funktion verfolgen und eine auf das Gemeinwohl ausgerichtete prosoziale Handlung sein. Andererseits kann Teilen eine Nutzungsstrategie sein, bei der u. a. Eigenwirtschaftlichkeit im Fokus steht und Ziele entlang von Kosten-NutzenAbwägungen definiert werden (Schlag und Rößger 2019, S. 11). Während Kosten für das Öffentliche, wie zuvor beschrieben, per Einheit anfallen und somit eine Dienstleistung verkörpern, müssen bei privatem Eigentum einmalig die gesamten Kosten des Kaufpreises getragen werden (,pay per use " und ,pay and use').

Die mediale Dimension von öffentlich beinhaltet die Zugänglichkeit und Erreichbarkeit von Informationen und Nachrichten. In Bezug auf die Öffentliche Mobilität heißt dies, dass Informationen zu den Ortsveränderungsmöglichkeiten für alle zugänglich und verständlich sein müssen. Das bedeutet insbesondere in Hinblick auf die neuen Mobilitätsdienstleistungen, dass Informationen und Zugang nicht ausschließlich über die digitalen Plattformen privater Betreiber erfolgen dürfen, um dem Gestaltungsanspruch Öffentlicher Mobilität gerecht zu werden. So gilt es auch hier, eine bedarfsgerechte Informationsbereitstellung zu berücksichtigen. 


\section{$4 \quad$ Rahmenbedingungen für Veränderungen von Mobilität und Verkehr}

Der Versuch, das Automobil als dominierendes Regime abzulösen, blickt auf eine lange Geschichte zurück. Immer wieder stand in den letzten zwanzig Jahren die Suche nach Alternativen oder eine Annäherung von MIV und ÖV im Fokus der Verkehrspolitik. Während der Fokus dabei insbesondere auf technischen Fortschritt und Effizienzsteigerung gelegt wurde, liegt dem aktuellen Planungsverständnis zugrunde, dass Veränderungen gleichermaßen auf Angebots- und auf Nachfrageseite erforderlich sind. Es bedarf entsprechend nicht nur einer Weiterentwicklung der physischen und technischen Infrastruktur, sondern auch einer Veränderung in Bezug auf die Mobilitätskultur, das Mobilitätsverhalten sowie das Handeln weiterer involvierter Akteure (Lyons 2012, S. 30). Transformationsprozesse können dabei durch sogenannte ,Treiber' angestoßen werden, wobei es sich um gesellschaftliche Trends, technische Entwicklungen, aber auch politische Maßnahmen handeln kann (Arnold et al. 2018, S. 52). Eine Reihe solcher Entwicklungen ist aktuell $\mathrm{zu}$ beobachten und für den dynamischen Wandel im Mobilitätssektor verantwortlich. Zu berücksichtigen ist allerdings, dass die Ausprägung der Entwicklungen in Abhängigkeit des jeweiligen Raumbezugs unterschiedlich ausfallen. Der häufig beschriebene Wandel bezieht sich zumeist auf den urbanen Kontext.

Entscheidend für die Öffentliche Mobilität ist auf gesellschaftlicher Ebene insbesondere der Trend zur Individualisierung, die mit einer Zunahme an Wahlund Entscheidungsmöglichkeiten einhergeht. Durch die entstandenen Freiheiten kommt es zu Ausdifferenzierungen in der privaten Lebensführung sowie der Berufswelt und letztendlich zu einer Pluralisierung von Lebensstilen. Im beruflichen Kontext nimmt die Dominanz traditioneller Arbeitsverhältnisse ab, sodass Arbeitskonditionen und -zeiten nicht mehr zu vereinheitlichen sind. Im Privatleben sind zudem weniger Eheschließungen, eine Abnahme der Geburtenzahlen und dementsprechend geringere Haushaltsgrößen zu verzeichnen. Einhergehend mit der Ausdehnung der Siedlungsflächen nimmt die Komplexität des Alltags zu und führt aufgrund der dispersen Verteilung relevanter Einrichtungen im Raum zu stark ausdifferenzierten Aktivitätsräumen (Rammler und Sauter-Servaes 2013, S. 25; Schneider 2018, S. 30 f.). Weitere zu nennende Effekte, die mit der Individualisierung in Verbindung gebracht werden, sind die sinkende Bedeutung des Automobils bei Teilen der jüngeren Generation, ein steigendes Umweltbewusstsein sowie ein erhöhtes Streben nach Flexibilität (Gouthier und Nennstiel 2018, S. 574). Anforderungen an die Mobilität gestalten sich dementsprechend 
sehr divers und variieren in Abhängigkeit der persönlichen Rahmenbedingungen. Insbesondere in Städten mit einem gut ausgebautem ÖPNV-System zeigt sich, welchen Stellenwert der ÖPNV einnehmen kann. Umso bedeutender ist es, das Image des öffentlichen Verkehrs als wenig flexibles ,Massentransportmittel ' zu ändern und mit der Gestaltung Öffentlicher Mobilität flexible, bedarfsgerechte und individuelle Mobilität zu stärken. Dieser, auf geänderte Anforderungen an die Mobilität zurückzuführende Veränderungsdruck, kann auch als „,demand pull“ bezeichnet werden (Schnieder und Gebhardt 2016, S. 2).

Demgegenüber stehen der technische Fortschritt sowie die Digitalisierung, die Möglichkeiten für eine Neugestaltung und Flexibilisierung der (öffentlichen) Mobilität auf Angebotsseite bieten. Durch das aktive Vorantreiben des Wandels wird auch vom sogenannten ,technology push" gesprochen (Schnieder und Gebhardt 2016, S. 2). Die Digitalisierung gilt als einer der zentralen Treiber für den Wandel im Mobilitätssektor und stößt als Megatrend zudem tiefgreifende Veränderungen in diversen anderen Sektoren an. Besonders umfassend ist der Einfluss auf die Mobilitätswelt, da sich die Digitalisierung sowohl auf Angebotsseite als auch auf Nachfrageseite auswirkt. Im Zentrum des Diskurses um Digitalisierung stehen jedoch überwiegend technische und wirtschaftlich relevante Entwicklungen, wohingegen Veränderungen und Potenziale auf Ebene potenzieller Nutzer*innen seltener beleuchtet werden. Technische und gesellschaftliche Entwicklungen sind dabei untrennbar ineinander verwoben und bedingen sich gegenseitig.

Da Digitalisierung als entscheidender Faktor und Basis für die neuen Mobilitätsdienstleistungen gilt, soll hier auf deren Bedeutung eingegangen werden. Im ursprünglichen Sinn wird dabei die Übertragung oder Übersetzung analoger Vorgänge und Handlungen aus der realen Welt in eine datengestützte, von Maschinen lesbare Form verstanden (Rees 2018, S. 5; Rürup und Jung 2017, S. 5). Häufig wird die Digitalisierung darüber hinaus mit den Effekten verbunden, die sie hervorruft. Sie ermöglicht und fördert die ständige Vernetzung von Menschen, Objekten und Orten über das Internet. Die Vernetzung geht mit der Nutzung von Informations- und Kommunikationstechnologien (IKT) einher, die mit der Sammlung von enormen Datenmengen (Big Data) verbunden wird, welche wiederum für verschiedene Zwecke verarbeitet und analysiert werden können. Gebündelt werden die Daten auf verschiedenen Plattformen, die als zentrale Verwaltungsstelle für Organisation und Aufbereitung von Informationen und Diensten gelten.

Die veränderten Rahmenbedingungen führen schließlich auch $\mathrm{zu}$ einem Wandel der Geschäftsmodelle in der Wirtschaft, bei denen vorwiegend Dienstleistungen im Fokus stehen. Für die Kund*innen bedeutet dies ein verändertes 
Konsumverhalten, das sich zunehmend vom Eigentum distanziert und den Fokus auf das Teilen von Gütern und die Inanspruchnahme Dienstleistungen setzt. Die Digitalisierung ist demnach Grundlage für einen Wandel hin zu der sogenannten Plattformökonomie oder auch zur Sharing-Economy (Rees 2018, S. 6; Lyons 2018, S. 4). Als entscheidende Voraussetzung und Schlüssel für die neuen Angebotsformen gilt die kontinuierlich wachsende Verbreitung des Smartphones, welches in der Regel den ständigen Zugang zu Informationen sowie zu Buchungs- und Zahlungsfunktionen der Dienstleistungen ermöglicht. In diesem Zusammenhang wird auch von der digitalen Mobilität gesprochen, die als ,die durch Technik unterstützte Bewegung in physischen und virtuellen Räumen“ (Schmoll et al. 2014, S. 7) definiert wird. Grundlage für die reibungslose Nutzung ist eine flächendeckend gute Netzqualität, wobei in Deutschland diesbezüglich regionale Unterschiede festzustellen sind und insbesondere im ländlichen Raum nach wie vor Defizite zu verzeichnen sind (Agora Verkehrswende 2017, S. 17 f.).

\section{$5 \quad$ Zur Bedeutung von ,neuer' Mobilität, Verkehrsdienstleistungen und neuen Mobilitätsdienstleistungen}

Im Zentrum der Debatte um die zukünftige Mobilität stehen sogenannte neue Mobilitätskonzepte, neue Mobilitätsangebote oder auch neue Mobilitätsdienstleistungen, auf die hier näher eingegangen werden soll. Die Verwendung der unterschiedlichen Begrifflichkeiten erfolgt dabei häufig synonym, eindeutige einheitliche Begriffsbestimmungen liegen dabei noch nicht vor.

Zunächst gilt es, das Adjektiv ,neu‘ zu beleuchten. Der Begriff kann in diesem Kontext mit Veränderung oder Weiterentwicklung verbunden werden und ist auf die zuvor beschriebene Digitalisierung als Treiber zurückzuführen. Gleichzeitig kann, neu' auch mit dem Begriff innovativ gleichgesetzt werden, wie es in der Debatte um die Transformation im Mobilitätssektor häufig geschieht. Innovationen können anhand ihres Bezugssystems unterteilt werden, sodass im Kontext von Mobilität und Verkehr zwischen Produktinnovationen, Nutzungsinnovationen und Systeminnovationen unterschieden wird.

Produktinnovationen begründen ihre Innovation im technischen Fortschritt, indem ein Produkt (z. B. ein Element der Infrastruktur oder ein Verkehrsmittel) modifiziert und effizienter gestaltet wird. Beispiele für Produktinnovationen sind Elektromobilität, autonomes Fahren, Effizienzsteigerungen im ÖPNV oder auch die kontrovers diskutierten E-Tretroller (Rammler und Sauter-Servaes 2013, S. 30). 
Nutzungsinnovationen beziehen sich auf den Verkehrsprozess und dessen Organisation. Dabei werden die bereits vorhandenen Produkte oder in diesem Fall Beförderungsmittel bezüglich ihrer Handlungsabläufe neu organisiert. Über die bereits erwähnte Plattformökonomie gestaltet sich der Zugang zu bereits bekannten Verkehrsmitteln neu. Im Zentrum steht die eigentumslose Nutzung bzw. die temporäre Übertragung von Besitz. Dabei verändert sich die ProduzentVerbraucher*innen-Beziehung oder auch die Anbieter-Nutzer*innen-Beziehung, indem Verkehr als Dienstleistung angeboten wird. Statt ein Fahrzeug einmalig zu kaufen und somit Eigentum zu erwerben, wird der temporäre Besitz, je Einheit (Distanz, Fahrt, Zeit) finanziert (statt ,pay and use', pay per use') (Rammler und Sauter-Servaes 2013, S. 33). Unter Nutzungsinnovationen fallen demnach auch die neuen Mobilitätsdienstleistungen, die im Anschluss ausführlich beleuchtet werden.

Bereits aus den vorangestellten Textpassagen wird deutlich, dass für die Gestaltung eines nachhaltigen und bedarfsgerechten Verkehrssystems ein ganzheitlicher und integrativer Ansatz notwendig ist (siehe den Beitrag von Schwedes in diesem Band). Als die Innovationsform mit der größten Transformationskraft gilt die Systeminnovation. Sie verknüpft die zuvor beschriebenen Produkt- und Nutzungsinnovationen und setzt zudem erhebliche Veränderungen in Bezug auf die Gesellschaft, die Infrastruktur (physisch und digital) und die Politik voraus. Aufgrund des gesamtsystemischen Ansatzes verspricht diese Form der Innovation die größten Potenziale, um ökologische und soziale Entlastungseffekte hervorzurufen und eine neue Mobilitätskultur zu etablieren. Durch die hohe Komplexität verzeichnet sie jedoch auch die geringste Umsetzungsgeschwindigkeit, da die Integration von öffentlichen, privatwirtschaftlichen, zivilgesellschaftlichen und verkehrspolitischen Interessen eine große Koordinationsherausforderung darstellt (Rammler 2014, S. 30).

\subsection{Neue Mobilitätsdienstleistungen als Nutzungsinnovation - Eine Differenzierung}

Zuvor wurden im Detail die hier zugrunde liegenden Definitionen von Verkehr und Mobilität beschrieben. Angewandt auf ,neue Mobilitätsdienstleistungen ' geht damit eine Differenzierung zwischen dem physischen und dem digitalen Angebot einher. So werden im weiteren Verlauf einerseits Verkehrsdienstleistungen beschrieben, die das physische Angebot, also die objektive Auswahl an Ortsveränderungsmöglichkeiten erweitern (Fahrdienste, Sharing-Angebote) und andererseits die digitalen Mobilitätsdienstleistungen, die zur besseren Wahrnehmung der 
objektiv verfügbaren Ortsveränderungsmöglichkeiten beitragen. Die neuen Verkehrsdienstleistungen werden in der Regel ausschließlich in Kombination mit digitalen Dienstleistungen zur Verfügung gestellt, während letztere auch für sich stehen können.

Dementsprechend wird im Rahmen dieses Beitrags als übergeordnete Begrifflichkeit auf die Bezeichnung, neue Mobilitätsdienstleistungen' zurückgegriffen, die entweder eine Kombination von Verkehrsdienstleistungen und digitalen Mobilitätsdienstleistungen umfasst aber auch eine rein digitale Dienstleistung sein kann. Aus der Verfügbarkeit digitaler Informationen und dem digitalen Zugang, resultiert für die Nutzer*innen eine gewisse Planbarkeit sowie die Möglichkeit, Angebote zu vergleichen, bevor sie in Anspruch genommen werden. Da die Dienstleistung je nach Bedarf erfolgt, bezieht sich „Mobilitätsdienstleistungen“ zunächst auf die Beweglichkeit, also die optionale Ortsveränderung und noch nicht auf die realisierte Ortsveränderung. Der ÖV wird im Gegensatz dazu als Verkehrsdienstleistung bezeichnet, da Wege aufgrund der festen Taktung nicht bedarfsorientiert, sondern nach Plan und unabhängig vom individuellen Fahrtwunsch erfolgen.

Wie zuvor beschrieben, wird im weiteren Verlauf zwischen dem physischen und dem digitalen Angebot unterschieden und neue Formen von Verkehrsdienstleistungen sowie digitale Mobilitätsdienstleistungen dargestellt.

\section{Verkehrsdienstleistungen}

Bei den Verkehrsdienstleistungen wird von zwei parallelen Entwicklungen gesprochen - von der Individualisierung des öffentlichen Kollektivverkehrs und von der Veröffentlichung des privaten Individualverkehrs. Diese Entwicklungen sind nicht neu, da flexible Bedienformen im ÖPNV (z. B. Rufbusse oder Anrufsammeltaxis) bereits seit den 1970er Jahren eingesetzt werden und Carsharing seinen Ursprung in den späten 1980ern hat (Rees 2018, S. 108 f.; Schwedes 2014b, S. 243). Dennoch kommt es nun augenscheinlich erst mit der Digitalisierung zu einer dynamischeren, marktgetriebenen Entwicklung und Verbreitung der flexiblen Angebote. Neu ist dabei der digitale Zugang. Nach wie vor lassen sich die Angebote in die stärker an dem ÖV angelehnten Fahrdienste unterteilen, bei der die Beförderung im Vordergrund steht sowie in Sharing-Dienste, die der Privatnutzung ähneln und Besitz auf Zeit ermöglichen.

Bei Fahrdiensten bezieht sich die Dienstleistung auf die Beförderung, ohne dass der oder die Nutzer*in selbst fahren muss. Die Buchung erfolgt innerhalb eines Geschäftsgebiets flexibel auf Abruf, weshalb hier auch von On-DemandVerkehren gesprochen wird. Nach Möglichkeit werden Fahrten mittels eines 
Algorithmus gebündelt, also ,gepoolt', um eine hohe Auslastung der Fahrzeuge zu gewährleisten. Während der Fahrt können entsprechend Umwege anfallen, um weitere Fahrgäste aufzunehmen (Rees 2018, S. 114). Sofern das Angebot von einem/kommerziellen Anbieter betrieben wird und ein/eine professionelle*r Fahrer*in eingesetzt wird, handelt es sich bei dem Dienst um Rideselling oder auch Ridepooling. Beispiele hierfür sind Moia in Hamburg (HVV/Volkswagen) oder der BerlKönig in Berlin (Berliner Verkehrsbetriebe/Daimler). Wenn die Fahrt explizit nicht geteilt wird und nur für einen einzelnen Nutzenden erfolgt, kann dieses Angebot als Ridehailing bezeichnet werden. Auch die klassische Mitfahrgelegenheit, bei der Privatpersonen einen Platz in ihrem Fahrzeug für eine bereits geplante und unabhängig von weiteren Mitfahrer*innen stattfindende Fahrt anbieten, kann als Verkehrsdienstleistung verstanden werden. Die Dienstleistung der zugehörigen Service-Plattform kann sich ausschließlich auf die Vermittlung beziehen oder aber auch zusätzlich Versicherungsschutz der Nutzenden garantieren. Das Konzept der Mitfahrgelegenheit, das auch als Ridesharing bezeichnet wird, ist eine Form des Peer-to-peer-Sharings, da hier ein Angebot zwischen Privatpersonen vermittelt wir. Ridesourcing beschreibt einen Fahrdienst, der zwar über eine kommerzielle Plattform angeboten wird, aber von privaten Fahrern mit ihren eigenen im Eigentum befindlichen Fahrzeugen angeboten wird. Das Personenbeförderungsgesetz (PBefG) legt in Deutschland fest, dass bei der entgeltlichen Beförderung von Personen ein Personenbeförderungsschein notwendig ist. Dementsprechend ist diese Form in Deutschland nicht zulässig, wie das Beispiel des Angebots UberPop zeigt. Als Konkurrenz gelten die ,neuen ' Fahrdienste nicht nur für den klassischen ÖPNV, sondern vor allem auch für die lokalen Taxi-Unternehmen, welche allerdings über den digitalen Zugang und die Möglichkeit, Fahrten zu teilen (z. B. FreeNow), auch in den Bereich neuer Mobilitätsdienstleistungen einzuordnen sind. Perspektivisch kann es sich bei allen beschriebenen Formen der Fahrdienste um ein Angebot mit autonomen Fahrzeugen handeln (Deutsch 2018, S. 259; Rees 2018, S. 113).

Sharing-Dienste implizieren die Veröffentlichung des privaten Individualverkehrs oder anders formuliert die Überführung des privaten Individualverkehrs in einen öffentlich zugänglichen. Die Sharing-Angebote beruhen dabei auf der zuvor beschriebenen Plattformökonomie und dem bereits thematisierten Konzept der eigentumslosen Nutzung. Die Dienstleistung umfasst in diesem Fall das zur Verfügung stellen des Fortbewegungsmittels für einen gewissen Zeitraum (Rees 2018, S. 107). Ein entscheidender Unterschied für die Nutzer*innen ist dabei, ob es sich um einen stationsgebundenen oder einen free-floating Sharing-Dienst handelt. Während stationsgebunden bedeutet, dass sowohl der Zugang als auch 
die Abgabe des Fahrzeugs an einem fest definierten Ort erfolgt, erlaubt das freefloating Sharing-Angebot das flexible Anmieten und Abstellen in einem vorgegebenen Nutzungsgebiet. Erst mit der Flexibilisierung mittels der digitalen Zugangsmöglichkeiten fand im Bereich der Sharing-Dienste ein enormer Zuwachs an Nutzenden und Angeboten statt (Rees 2018, S. 109; Giesel und Nobis 2016, S. 223). Zwar steht im Fokus häufig der motorisierte Individualverkehr in Form unterschiedlicher Car-Sharing-Angebote, darüber hinaus können aber auch weitere (Individual-) Verkehrsmittel, wie Fahrräder, Lastenräder, E-Roller oder E-Tretroller, über Plattformen als Dienstleistung in Anspruch genommen werden. Im Gegensatz zu den Fahrdiensten wird bei Sharing-Diensten die eigene Fahraktivität erforderlich. Das bedeutet, dass für den Zugang des Angebots der Besitz einer Fahrzeugerlaubnis, die Kenntnisse über die Nutzung des jeweils spezifischen Fahrzeugs und der Straßenverkehrsregeln sowie das Vorhandensein der körperlichen und psychischen Voraussetzungen notwendig ist. Das angemietete Verkehrsmittel wird zum Zeitpunkt der Nutzung nicht geteilt, sodass der temporäre Besitzzustand verstärkt wird und den Eindruck von Privatheit, trotz gemeinschaftlicher Nutzung unterstützt. Abb. 1 liefert einen zusammenfassenden Überblick der geschilderten Ausführungen zu den Verkehrsdienstleistungen.

\section{Digitale Mobilitätsdienstleistungen und Mobility-as-a-Service (MaaS)}

Wie eingangs erwähnt, werden neben den Verkehrsdienstleistungen als physisches Angebot auch digitale Mobilitätsdienstleistungen angeboten, die sich mit Information, Organisation, Buchung und der Koordination von Ortsveränderungsmöglichkeiten befassen. Ausgehend von der Digitalisierung, ist das zentrale Instrument eine digitale Plattform - zumeist in Form einer App auf dem Smartphone. Durch die Nutzung standortbezogener Daten kann, jeweils auf die individuellen Bedürfnisse abgestimmt, die subjektiv beste Option für die Ortsveränderung gewählt werden.

Monomodale (Informations-)Dienstleistungen beziehen sich dabei ausschließlich auf ein Verkehrsmittel und unterstützen Nutzer*innen bei der Planung von Fahrten sowie während einer laufenden Fahrt (pre-trip-/on-trip-services). Entsprechende Anwendungen existieren in vielfacher Ausführung für alle verfügbaren Ortsveränderungsmöglichkeiten und variieren dabei von der reinen Information (Navigation, Auskunft über Abfahrten) über Reservierungs-, Buchungs- und Bezahloptionen oder andere mobilitätsbezogene Services (z. B. Parkplatz-App) (Lanzendorf und Hebsaker 2017, S. 141; Gouthier und Nennstiel 2018, S. 579). Der Service kann dementsprechend unterschiedliche Aktivitäten, bezogen auf ein Verkehrsmittel, vereinfachen. Auch bei den zuvor beschriebenen 


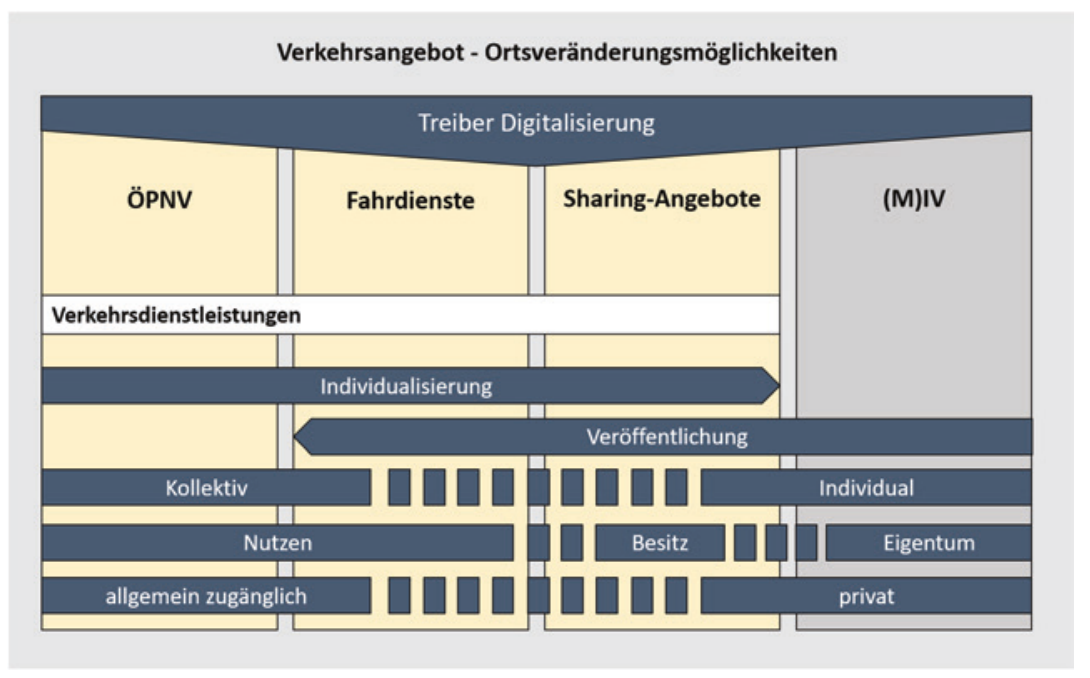

Abb. 1 Erweiterung des Verkehrsangebots durch Verkehrsdienstleistungen. (Quelle: eigene Darstellung)

Verkehrsdienstleistungen, Sharing- und Fahrdiensten, eröffnet sich der öffentliche Zugang erst über die ergänzende digitale Plattform, die über zeitliche und räumliche Verfügbarkeit informiert und die Buchung organisiert. Mittels des Service kann eine sogenannte vertikale Integration von Angeboten erfolgen, bei der unterschiedliche Anbietende/Formen eines Verkehrsangebotes zusammengefasst und integriert dargestellt werden.

Beispielsweise kann in einer ÖV-App der Informations- und Buchungsdienst übergreifend für den Schienenpersonennahverkehr und den öffentlichen Straßenpersonennahverkehr erfolgen oder bei einer Car-Sharing-App können verschiedene Anbietende angezeigt werden. Eine Herausforderung für die Nutzer*innen stellt die Fülle unterschiedlicher Informations- und Organisationsplattformen dar, weil die einzelnen Angebote für sich stehen. Unattraktiv ist das für die Kund*innen deswegen, weil sie sich bei der Planung sowohl für ein Verkehrsangebot als auch für einen Anbieter entscheiden müssen oder eigenständig Vergleiche zu Verfügbarkeiten und der für die individuellen Bedürfnisse besten Option anstellen müssen. Dies kann zu Unübersichtlichkeit und somit zur eingeschränkten subjektiven Wahrnehmung der vielfältigen Angebote führen. 
Multimodale (Informations-)Dienstleistungen greifen diese Problematik auf, indem sie den Anspruch haben, auf einer Plattform unterschiedliche physische Mobilitätsdienstleistungen verkehrsmittel- und anbieterübergreifend zu integrieren (Lanzendorf und Hebsaker 2017, S. 138). Entsprechend kann hier von der digitalen Integration der allgemein zugänglichen Ortsveränderungsmöglichkeiten gesprochen werden. In der Debatte um neue Mobilität etabliert sich in diesem Kontext zunehmend die Bezeichnung Mobility-as-a-service (MaaS) für das Konzept, was per Definition mit einer multimodalen Plattform gleichgesetzt werden kann. Für den weiteren Verlauf wird in diesem Beitrag auf die Bezeichnung MaaS zurückgegriffen. Begriffsdefinitionen variieren stark, sodass MaaS unterschiedliche Bedeutungen haben kann. Es gilt, MaaS als Plattform von der Begrifflichkeit Mobility Service, also Mobilitätsdienstleistung, klar zu trennen. Entgegen der hier verwendeten Definition wird MaaS häufig als Überbegriff für neue Mobilitätsdienstleistungen verwendet.

Die Voraussetzung für MaaS liegt dabei in einem vielfältigen Angebot unterschiedlicher Mobilitätsdienstleistungen sowie einer starken und funktionierenden digitalen Infrastruktur (Kamargianni und Matyas 2017, S. 12). Immer wieder wird in der Literatur als zentrales Charakteristikum der nachfragezentrierte, nutzer*innenorientierte Ansatz betont (Jittrapirom et al. 2017, S. 16; Giesecke et al. 2016, S. 1; Kamargianni und Matyas 2017, S. 12). Der Vorteil für die Kund*innen besteht darin, dass ihnen mit der Anmeldung bzw. dem Zugang zu einer einzigen Plattform eine Art ,Mobilitätspaket` zur Verfügung gestellt wird, bei dem nicht nur Informationen, sondern auch Buchungs- und Bezahloptionen zentral durch einen MaaS-Betreiber (MaaS-operator) verwaltet werden. Somit wird die Kombination und flexible Wahl der Verkehrsmittel möglich (Hensher und Mulley 2020, S. 2). In der Umsetzung stellt die multimodale und anbieterübergreifende Integration zu einem Mobilitäts-Angebot eine Herausforderung dar, denn für einen ganzheitlichen Ansatz werden Kooperationen zwischen öffentlichen und privaten Akteuren unterschiedlicher Ebenen (globale wie lokale) nötig. Für eine erfolgreiche Umsetzung ist es essenziell, dass auf der Plattform für die Kund*innen möglichst alle verfügbaren Ortsveränderungsmöglichkeiten digital sichtbar sind und zur Verfügung gestellt werden. Zwar ist die Auswirkung von MaaS auf das Nutzer*innenverhalten bislang wenig erforscht, dennoch wird ein großes Potenzial für die Gestaltung einer bedarfsgerechten und nachhaltigen Mobilität gesehen (Matyas und Kamargianni 2018, S. 2). Im späteren Verlauf dieses Kapitels werden Anforderungen an MaaS als Instrument des Mobilitätsmanagements vertiefend beleuchtet und Perspektiven für die Verkehrsplanung aufgezeigt.

In Abb. 2 wird die Differenzierung der digitalen Mobilitätsdienstleistungen zusammenfassend und schematisch aufgearbeitet. Hierbei handelt es sich um 


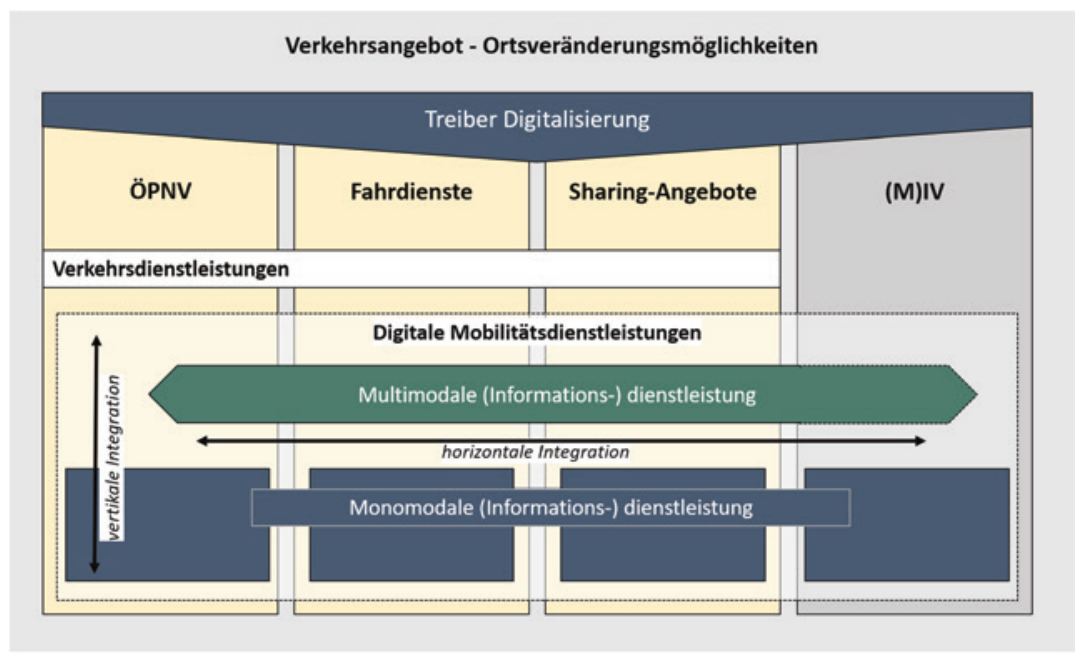

Abb. 2 Erweiterung der subjektiven Ortsveränderungsmöglichkeiten durch digitale Mobilitätsdienstleistungen. (Quelle: eigene Darstellung)

eine ideelle Darstellung, wissend, dass die neuen Mobilitätsdienstleistungen nicht flächendeckend verfügbar sind und eine ganzheitliche horizontale Integration bislang wenig erfolgreich ist.

Nachdem nun Verkehrsdienstleistungen, die die objektiven Ortsveränderungsmöglichkeiten erweitern sowie die digitalen Mobilitätsdienstleistungen, die deren subjektive Wahrnehmung beeinflussen, beleuchtet wurden, kann für neue Mobilitätsdienstleistungen oder -dienste folgende Definition festgehalten werden: „Neue Mobilitätsdienstleistungen sind auf den individuellen Mobilitätsbedarf abgestimmte Services digitaler oder physischer Natur, die (die subjektive Wahrnehmung von) Ortsveränderungsmöglichkeiten für einen begrenzten Zeitraum gegen eine Bezahlung erweitern. “

\subsection{Die Positionen der Akteure}

Die veränderten Rahmenbedingungen, die Produkt- und vor allem die Nutzungsinnovationen in Form neuer Mobilitätsdienstleistungen fördern die vielfach anvisierte Annäherung von MIV und ÖV. Für die Nutzer*innen ist diese Entwicklung positiv, da ihnen mehr und flexiblere Ortsveränderungsmöglichkeiten 
zur Verfügung stehen und auch ohne den privaten Pkw individuelle und bedarfsgerechte Mobilität möglich scheint. Unter gewissen Voraussetzungen stellen sie einen Anreiz für einen Paradigmenwechsel weg von der verkehrsmittel- und besitzorientierten Mobilität hin zu einer nutzungsorientierten Mobilität dar. Durch das diversifizierte Angebot kann multimodales Mobilitätsverhalten erleichtert und der Umweltverbund gefördert werden.

Bislang führten Annäherungsversuche seitens der ÖV-Anbieter aufgrund von strukturellen Blockaden und wirtschaftlichen Risiken zu wenig Erfolg. Die gewünschte Flexibilisierung des ÖV und Veröffentlichung des Individualverkehrs scheint nun zu gelingen - jedoch überwiegend durch marktgetriebene Angebote der Privatwirtschaft mit geringem Einfluss und Steuerung seitens der Politik und der Kommunen. Die ursprüngliche Aufgabe, Verkehrsdienstleistungen bereitzustellen, liegt bei den ÖV-Unternehmen. Durch die von der Digitalisierung geprägten Nutzungskonzepte ergibt sich eine Diversifizierung der Anbieter. Es entsteht ein neues organisationales Feld, in dem Akteure mit sehr unterschiedlichen Zielsetzungen und Voraussetzungen sich gemeinsam auf neue Rahmenbedingungen, Dynamiken, Netzwerke, Normen und Werte einstellen müssen (Meyer 2014, S. 170).

Der öffentlichen Hand stehen dabei private Unternehmen aus der Automobil-, Energie- oder IT-Branche gegenüber. Nahezu alle großen Automobilkonzerne bieten mittlerweile eine Form von Mobilitätsdienstleistungen an, wobei häufig die Produktinnovationen (z. B. Elektrofahrzeuge) präsentiert werden. Im Bereich der privaten Akteure ergibt sich ein breites Spektrum von etablierten (globalen) Konzernen, wie Daimler, BMW oder Google, bis hin zu kleinen (lokalen) StartUps, wodurch das Feld der traditionellen Akteure um neue Akteure erweitert wird (Karlsson et al. 2020, S. 286; Lanzendorf und Hebsaker 2017, S. 137). Dabei kann bezüglich der Zuständigkeiten innerhalb des Feldes keine eindeutige Zuordnung mehr zu den ursprünglichen Tätigkeitsbereichen der Anbietenden vorgenommen werden. So können zum Beispiel ÖV-Anbieter auch Car-Sharing anbieten (z. B. flinkster von der DB) oder Automobilkonzerne flexible Fahrdienste bereitstellen (Moia von VW). Es zeigt sich, dass alle Anbietenden im Bereich der neuen Mobilitätsdienstleistungen (sowohl in Bezug auf digitale Mobilitätsdienstleistungen als auch Verkehrsdienstleistungen) das Ziel haben, ein möglichst breites Portfolio zu entwickeln. Häufig werden von einem Anbietenden Fahrdienste, Sharing-Dienste, sowie multimodale (Informations-)Plattformen gleichzeitig angeboten (z. B. Daimler).

Bislang sind Rollenverteilung und Verantwortung der Akteure in Bezug auf die Entwicklung einer nachhaltigen bedarfsgerechten Mobilität noch nicht klar definiert. 
Relevante Akteure im Feld der (öffentlichen) Mobilität können in unterschiedliche Ebenen untergliedert werden. Auf Makroebene ist die Bundesregierung dafür verantwortlich, den rechtlichen Rahmen festzulegen, Zielsetzungen sowie die Finanzierung festzulegen. Auf Meso-Ebene sind Regional- und Kommunalpolitik für die Ausgestaltung konkreter Ziele und Regulierungen zuständig. Zudem müssen hier sowohl die öffentlichen als auch die privaten Anbieter koordiniert werden und sich gegenseitig, aber auch mit der Politik abstimmen. Auf Mikroebene finden sich letztendlich die Nutzer*innen wieder, die mit ihren Einstellungen, Normen und jeweiligen Voraussetzungen über Akzeptanz und Erfolg der neuen Mobilitätsdienstleistungen bestimmen. Für die zukünftige Gestaltung Öffentlicher Mobilität ist es von entscheidender Bedeutung, klare Verantwortlichkeiten und Aufgaben zu definieren (Karlsson et al. 2020, S. 285). Denn bereits das Politikfeld des ÖV ist geprägt von undeutlichen Verantwortlichkeiten (Karl 2008, S. 30).

\subsection{Kritik an neuen Mobilitätsdienstleistungen}

Kritik an den neuen Mobilitätsdienstleistungen ist häufig auf die stark wirtschaftlich handelnden Betreiber zurück zu führen, wobei sich Gemeinwohl-Interessen und wirtschaftliche Interessen gegenüberstehen. Da die (privatwirtschaftlich betriebenen) Angebote ausschließlich auf Effizienz und maximalen Profit ausgelegt sind, treten am Gemeinwohl orientierte Faktoren der allgemeinen Zugänglichkeit häufig in den Hintergrund, sodass etwa die räumliche Verfügbarkeit stark begrenzt ist (Docherty et al. 2018, S. 119). Oft sind Fahrdienste und SharingDienste lediglich auf die Stadtzentren von Metropolen mit mehr als 500.000 Einwohner*innen beschränkt, obwohl hier bereits ein gutes ÖPNV-Netz besteht. Selten werden die Angebote in Abstimmung auf das lokale ÖV-Netz etabliert, sodass hier eher Konkurrenz entsteht und sogar von der Kannibalisierung des ÖV gesprochen wird (Deutsch 2018, S. 258; Lanzendorf und Hebsaker 2017, S. $146 \mathrm{f}$.). Die bislang stark wirtschaftsgetriebenen neuen Mobilitätsdienstleistungen haben zur Folge, dass demnach statt einer angestrebten Integration und der Ergänzung von Verkehrsmitteln vorwiegend Konkurrenz zwischen den Angeboten und Akteuren besteht. Einerseits entsteht durch den Wettbewerb eine Vielzahl an Auswahlmöglichkeiten, dennoch mangelt es an einem ganzheitlichen und integrierten Gesamtkonzept. Durch Dopplung von Angebotsformen entstehen Ineffizienzen im Verkehrssystem, zudem variieren die Nutzungskonditionen (Kosten, Zugang, räumliche Verfügbarkeit usw.), was wiederum wenig nutzer*innenfreundlich ist und $\mathrm{zu}$ Unübersichtlichkeit führt. Indem es in 
der Umsetzung noch daran scheitert eine übergreifende Plattform anzubieten, kann die große Zahl unterschiedlicher digitaler Zugangsplattformen zu einer verminderten Wahrnehmung aus Kund*innensicht führen. Zwar sind bereits erste Kooperationen von Anbietern erkennbar (z. B. Jelbi oder Sharenow), dennoch fehlt es an einem leitenden Gesamtansatz, insbesondere von Seiten staatlicher Akteure. Für ein ganzheitliches Konzept braucht es klare Regelungen seitens der Politik, um für die Nutzer*innen ein bestmögliches öffentliches Mobilitätsangebot zu schaffen und somit eine umweltfreundliche Mobilität zu fördern.

\section{4 Öffentliche Mobilität als Systeminnovation}

Mit dem Ziel einen Wandel in der Planungskultur zu gestalten, bei der statt der Ausgestaltung und Planung einzelner Verkehrsmittel ein ganzheitlicher integrierter Ansatz verfolgt wird, kann die Gestaltung Öffentlicher Mobilität als anzustrebende Systeminnovation beschrieben werden (Docherty et al. 2018, S. 118). Basierend auf der Digitalisierung ergeben sich für die Öffentliche Mobilität verschiedene Perspektiven, da sich zum einen die objektiven Ortsveränderungsmöglichkeiten durch neue Angebotsformen erweitern und zum anderen die subjektive Wahrnehmung digitale Plattformen einbeziehen kann. Im Hinblick auf die allgemeine Zugänglichkeit gilt es klar zu analysieren, inwiefern ein Angebot tatsächlich zur Öffentlichen Mobilität gehört. Es wird zwar nicht vorausgesetzt, dass der Anbietende öffentlich sein muss, aber dennoch muss der Mobilitätsdienst, den er zur Verfügung stellt, den Anforderungen einer Öffentlichen Mobilität gerecht werden. Für die Gestaltung Öffentlicher Mobilität seitens der Verkehrspolitik besteht die Notwendigkeit klarer Zieldefinitionen und Vorgaben (normative Integration). Vor diesem Hintergrund wird nachstehend das normative Verständnis, das Öffentliche Mobilität impliziert, zusammenfassend dargestellt.

Bereits dem anfangs definierten Begriff des ,öffentlichen' liegen normative Vorstellungen zugrunde, sodass eine rein empirisch-analytische, wertfreie Verwendung grundsätzlich ausgeschlossen ist. ,Öffentlich ‘ kann somit als ,normatives Postulat', also als ein anzustrebender Zustand beschrieben werden (Jarren und Donges 2011, S. 96). Öffentliche Mobilität gilt als Kompromiss zwischen individueller Automobilität und dem öffentlichen Verkehr, wobei zu betonen ist, dass der traditionelle ÖV an sich auch ein fester Bestandteil Öffentlicher Mobilität ist. Allerdings erfährt der konventionelle ÖV im Rahmen der Systeminnovation vor allem aus Sicht der Nutzer*innen eine neue Qualität und kann bedarfsgerechter gestaltet werden. Die normative Zielvorstellung kann anhand der jeweils positiven Charakteristika der Verkehrsmittel (MIV und 
ÖV) hergeleitet werden. Das bedeutet einerseits: Flexibilität, Individualität, Unabhängigkeit und Komfort für die Nutzer*innen und andererseits Effizienz, Umweltfreundlichkeit und Sicherung der Mobilität auf der Angebotsseite. Orientiert am Leitbild der Integrierten Verkehrsplanung ist ein weiteres Ziel eine anbieter- und verkehrsmittelübergreifende Integration, die Inter- und Multimodalität fördert. Letztendlich bedeutet dies für die Planung, die Kooperation und Koordination verschiedener Akteure $\mathrm{zu}$ initiieren und $\mathrm{zu}$ managen, kund*innenorientierte und flexible Planung, die Bereitstellung (digitaler) Infrastruktur und die entsprechende rechtliche Rahmensetzung. Zudem ist es wichtig, dass die Ziele auf kommunaler Ebene weiter ausdifferenziert werden. Für die Ausgestaltung der neuen Mobilitätsdienstleistungen werden detaillierte Absprachen zwischen Kommunen und Anbietenden notwendig, um Ansprüchen an die allgemeine Zugänglichkeit gerecht zu werden (räumlich, sozial, materiell und medial). Begleitend zu Pull-Maßnahmen im Rahmen der Verbesserung Öffentlicher Mobilität, müssen Push-Maßnahmen eingesetzt werden, welche die Attraktivität des MIV vermindern, denn erst durch die Kombination von Pushund Pull-Maßnahmen ergibt sich eine Veränderung im Mobilitätsverhalten.

\section{Fazit: Gestaltungsperspektiven Öffentlicher Mobilität im Kontext mobilitätsbezogener Transformationsprozesse}

Die beschriebenen Veränderungen und Entwicklungen im Kontext von Mobilität und Verkehr eröffnen neue Gestaltungsmöglichkeiten im Hinblick auf die zukünftige Öffentliche Mobilität. Veränderte Rahmenbedingungen erfordern und ermöglichen zugleich eine bedarfsgerechte, ökologisch verträgliche und ökonomisch effiziente Öffentliche Mobilität, deren Planung sich an den Bedürfnissen der Nutzer*innen orientiert. Unter Betrachtung der Gestaltungsdimensionen der Integrierten Verkehrsplanung lassen sich folgende Ansätze festhalten.

Bezogen auf die Infrastruktur ist die Erweiterung um eine digitale Dimension notwendig, bei der unter anderem der Ausbau hin zu zuverlässigen und flächendeckenden Datennetzen essenziell ist. Einerseits kann so mittels Echtzeit-Daten der Zugang zum jeweiligen Verkehrsangebot einfacher gestaltet werden, anderseits eröffnen sich, basierend auf der Analyse gesammelter Datensätze, Chancen für Anbieter*innen, Verkehrsplanung und Forschung, flexibler auf die Bedürfnisse der Nutzer*innen einzugehen und das Verkehrsangebot effizienter zu gestalten. So kann beispielsweise die Auslastung öffentlicher Verkehrsmittel erhöht werden (Hasse et al. 2017, S. 8). Die Herausforderung liegt dabei in der 
verlässlichen Verfügbarkeit von Daten, dem Datenschutz sowie der Anforderung, Anpassungen der Infrastruktur auch flexibel und kurzfristig umsetzen zu können. Neben der digitalen Infrastruktur können zudem verknüpfende Elemente in Form von Mobilitätsstationen die Sichtbarkeit neuer Mobilitätsdienstleistungen erhöhen. In Berlin setzt die BVG sogenannte Mobilitäts-Hubs in Kombination mit der App Jelbi um und bündelt unterschiedliche Verkehrsangebote an einem Punkt (BBSR 2015, S. 6; Stadtraum 2019). Durch die ständige Verfügbarkeit von aktuellen und standortbezogenen Informationen über das Smartphone eröffnen sich bezogen auf die Verkehrsmittelwahl für den Verkehrsprozess flexiblere Verhaltensweisen, den Zeitpunkt sowie den Zugangspunkt der Ortsveränderung. Daraus ergibt sich eine gewisse Planungsfreiheit und zugleich -sicherheit, die zu einem erhöhten Komfort- und Autonomiegefühl führen kann. Durch Informations- und Kommunikationsplattformen entsteht zudem die Möglichkeit, Verkehrsmittel flexibler nutzen und besser kombinieren zu können, wodurch inter- und multimodales Mobilitätsverhalten erleichtert wird.

Bezogen auf die Mobilität und auf das Mobilitätsverhalten ergeben sich auch auf Ebene der Nutzer*innen Möglichkeiten, die subjektive Wahrnehmung von Ortsveränderungsmöglichkeiten zu optimieren. Im Rahmen von Maßnahmen des Mobilitätsmanagements eröffnen sich für die Verkehrspolitik ferner Handlungsspielräume in Form digitaler Organisations- und Informationsmaßnahmen. Allerdings entstehen hier neue Herausforderungen, über die verfügbaren Mobilitätsoptionen adäquat zu informieren und diese zu managen. Entscheidend ist dabei auch, dass nicht nur wirtschaftlich getriebene Angebote digital präsent sind, sondern auch für die Öffentliche Mobilität eine entsprechende Plattform geschaffen wird. Die digitale Zugänglichkeit nimmt dabei an Bedeutung zu, denn auf dem mittlerweile stark digitalisierten Markt ist es für Angebote jenseits von Apps und Plattformen schwer, wahrgenommen zu werden (Rees 2018, S. 7).

Zur Gestaltung einer bedarfsgerechten und umweltfreundlichen Mobilität gibt es mittlerweile viele Entwicklungen und Innovationen, die das physische und digitale Angebot an Mobilitätsdienstleistungen erweitern. Während Bund und Länder die Voraussetzungen und Rahmenbedingungen für die Förderung Öffentlicher Mobilität liefern müssen, liegt die Aufgabe der Umsetzung, Steuerung und Koordination der vorhandenen Angebote hinsichtlich einer Transformation lokaler Verkehrssysteme in der Verantwortung der Kommunen. Essenziell ist hierbei, ein Verständnis davon zu entwickeln, unter welchen Umständen ein Angebot als ,öffentlich' bzw. allgemein zugänglich gilt und welche rechtlichen Rahmenbedingungen dafür gesetzt werden können. Um entsprechend des Leitbilds der Integrierten Verkehrsplanung die neuen Angebote zu bündeln und kombinierbar zu machen, bietet sich das Mobilitätsmanagement als Instrument für die 
Verkehrspolitik an. Für potenzielle Nutzer*innen kann die Attraktivität gesteigert werden, indem mittels des Einsatzes von MaaS, als multimodale Informationsplattform, die Wahrnehmung verbessert wird, ein besserer Überblick vermittelt wird und der Zugang sowie die Kombinierbarkeit der Angebote vereinfacht werden. Aus planerischer Sicht ergeben sich durch den dynamischen Austausch mit den Kund*innen Möglichkeiten, das Mobilitätsverhalten mit weiteren spezifischen Maßnahmen des Mobilitätsmanagements zu beeinflussen (z. B. Vergleich von $\mathrm{CO}_{2}$-Emissionen, Boni bei grünen Wegen, Mobilitätsbudget usw.). Denkbar ist zudem eine Ausdifferenzierung des Angebots durch maßgeschneiderte Paketbuchungen mit unterschiedlichen Komfort-Stufen. Wichtig ist es, bei der Gestaltung von Mobility-as-a-service als Instrument des Mobilitätsmanagements darauf zu achten, dass soziale Exklusion vermieden wird (Jittrapirom et al. 2017, S. 17; Giesecke et al. 2016, S. 6).

Während MaaS aus Kund*innensicht eine Informations- und Buchungsplattform darstellt, kann es für die Politik auch eine Plattform sein, um unterschiedliche Anbietende zu koordinieren und zu verwalten. Die Voraussetzung für ein effizientes und erfolgreiches Angebot liegt vor allem in der Kooperation und Abstimmung privater und öffentlicher Akteure, wobei die Kommunikation sowohl zwischen den unterschiedlichen Akteuren als auch auf unterschiedlichen Ebenen notwendig ist (Jittrapirom et al. 2017, S. 16). Für eine einheitliche und gemeinsam entwickelte Strategie, unter Berücksichtigung der unterschiedlichen Interessen, ist es notwendig, neue Akteursnetzwerke aufzubauen, welche die Zusammenarbeit zwischen Politik, Verwaltung, Privatwirtschaft und Zivilgesellschaft ermöglichen (politische Integration). Dabei müssen Akteure die Bereitschaft zeigen, neue Rollen und Aufgaben zu übernehmen. Um Potenziale von MaaS ausschöpfen zu können, bedarf es zudem der Anpassung der Gesetzgebung, wobei die Herausforderung ist, einen Kompromiss zwischen Regulierung und Spielraum für Innovationen zu finden. Veraltete Rechtsvorschriften können ein Hindernis für die Entwicklung von MaaS sein (Karlsson et al. 2020, S. 292). Ein Beispiel dafür, wie neue Mobilitätsdienstleistungen auch in Gesetzestexten verankert werden können, liefert das Mobilitätsgesetz in Berlin (siehe den Beitrag von Kirchner in diesem Band).

Für das Zusammenführen und die Koordination der unterschiedlichen Interessen, die hinter den Angeboten stehen, braucht es einen zentralen Akteur, der die Aufgabe des Mobilitätsmanagements übernimmt und dabei die normativen Ziele für eine Öffentliche Mobilität berücksichtigt. Bislang stammen Betreiber von MaaS vorwiegend aus dem privatwirtschaftlichen Sektor. Vieles 


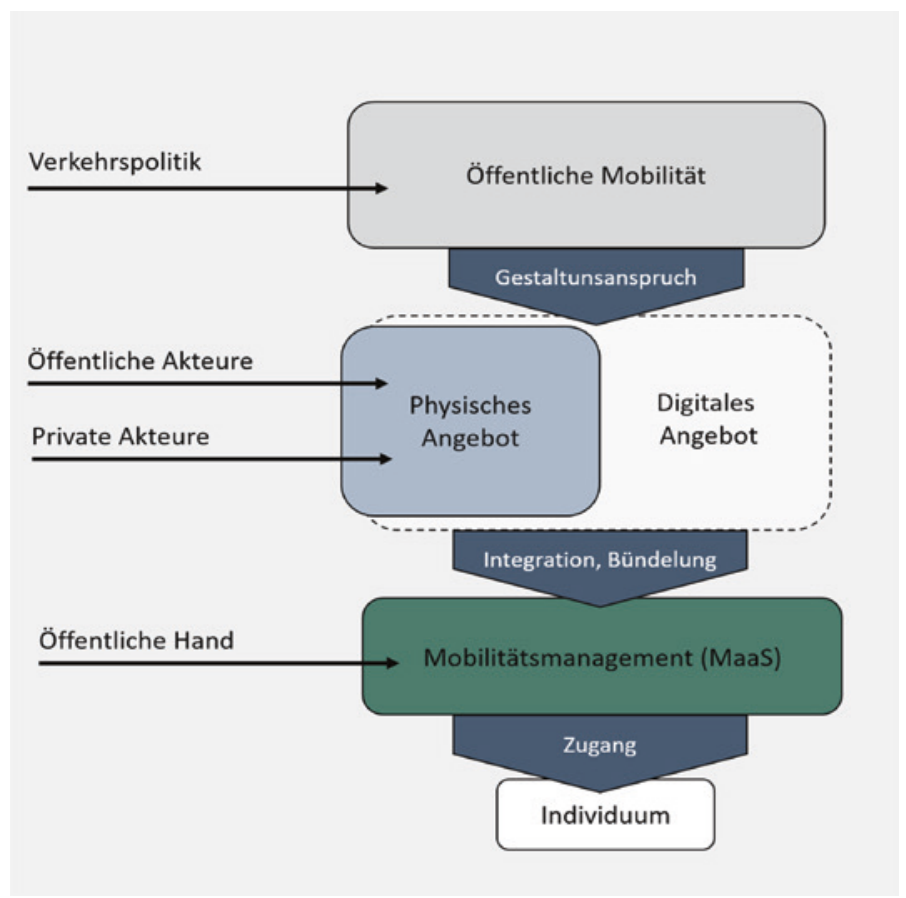

Abb. 3 Die Funktion von MaaS als Instrument des Mobilitätsmanagements. (Quelle: eigene Darstellung)

spricht dafür, dass die Aufgabe des ,Mobilitätsmanagers' seitens der Kommunen oder von öffentlichen Verkehrsanbietern übernommen werden müsste, da so eine klare Kontrolle über Regulierung und Gestaltung des öffentlichen Mobilitätsangebots möglich ist. Abb. 3 zeigt, die potenzielle Funktion von öffentlichem MaaS als Instrument des Mobilitätsmanagements.

Da das Konzept noch neu ist und wenig Erfahrungen $\mathrm{zu}$ der öffentlichen Form von MaaS vorliegen, bedarf es einer weiterführenden Untersuchung zu den Gestaltungsmöglichkeiten. Die Analyse von Pilotprojekten kann Aufschluss über die Anforderungen an MaaS als verkehrspolitisches Instrument zur Koordinierung Öffentlicher Mobilität geben. 


\section{Literatur}

Allgemeiner Deutscher Automobil-Club e. V. (ADAC). 2020. Zukunftsfähige öffentliche Mobilität außerhalb von Ballungsräumen. Konzeption einer Angebots- und Organisationsmodernisierung. Ergebnisbericht. https://assets.adac.de/image/upload/ v1581494746/ADAC-eV/KOR/Text/PDF/zukunftsfaehige-oeffentliche-mobilitaetausserhalb-von-ballungsraeumen_ADAC_Studie_kkr955.pdf. Zugegriffen: 4. April 2020.

Agora Verkehrswende, Hrsg. 2017. Mit der Verkehrswende die Mobilität von morgen sichern. 12 Thesen zur Verkehrswende (Kurzfassung).

Arnold, Annika, J. Schippl und S. Wassermann. 2018. Von der Nische in den Mainstream? Über Akteure, Angebote und das Diffusionspotential von Mobility as a Service. Stuttgarter Beiträge zur Risiko- und Nachhaltigkeitsforschung. 32.

Becker, Udo J. 2001. Was ist nachhaltige Mobilität? https://material.htlwien10.at/uzsb/ zusatzmaterial/nachhalt_mobil.pdf. Zugegriffen: 10. Mai 2017.

Bundesinstitut für Bau-, Stadt- und Raumforschung (BBSR), Hrsg. 2015. Neue Mobilitätsformen, Mobilitätsstationen und Stadtgestalt. Kommunale Handlungsansätze zur Unterstützung neuer Mobilitätsformen durch die Berücksichtigung gestalterischer Aspekte. Bonn.

Bundesministerium für Umwelt, Naturschutz und nukleare Sicherheit (BMU). 2016. Klimaschutzplan 2050. Klimaschutzpolitische Grundsätze und Ziele der Bundesregierung. https://www.bmu.de/fileadmin/Daten_BMU/Download_PDF/Klimaschutz/ klimaschutzplan_2050_bf.pdf. Zugegriffen: 6. April 2020.

Canzler, Weert. 2012. Automobilität und Gesellschaft: Zur Verortung einer sozialwissenschaftlichen Mobilitätsforschung. Soziale Welt 63(4): 317-337.

Canzler, Weert. 2016. The paradoxical nature of automobility. In: W. Canzler, S. Kesselring und V. Kaufmann, Hrsg. Tracing Mobilities. Towards a Cosmopolitan Perspective, 105-118. London: Taylor and Francis.

Deffner, Jutta, T. Hefter und K. Götz. 2014. Multioptionalität auf dem Vormarsch? Veränderte Mobilitätswünsche und technische Innovationen als neue Potenziale für einen multimodalen Öffentlichen Verkehr. In: O. Schwedes, Hrsg. Öffentliche Mobilität. Perspektiven für eine nachhaltige Verkehrsentwicklung. 2., aktualisierte und erw. Aufl., 201-227. Wiesbaden: Springer VS.

Deutsch, Volker. 2018. Autonome Betriebsformen in einem erweiterten ÖPNV-Markt. Perspektive autonomer und bedarfsgesteuerter Betriebsformen in einem erweiterten ÖPNV-Markt. Straßenverkehrstechnik 62(4): 258-267.

Docherty, Iain, G. Marsden und J. Anable. 2018. The governance of smart mobility. Transportation Research Part A: Policy and Practice 115: 114-125. https://doi.org/10.1016/j. tra.2017.09.012..

Dudenredaktion. 2020. „öffentlich“ auf Duden online. https://www.duden.de/rechtschreibung/oeffentlich. Zugegriffen: 13. Mai 2020.

Dziekan, Katrin und M. Zistel. 2018. Öffentlicher Verkehr. In: O. Schwedes, Hrsg. Verkehrspolitik, 347-372: Springer Fachmedien Wiesbaden.

Fraedrich, Eva und B. Lenz. 2015. Vom (Mit-) Fahren: autonomes Fahren und Autonutzung. In: M. Maurer, J. C. Gerdes, B. Lenz und H. Winner, Hrsg. Autonomes Fahren. Technische, rechtliche und gesellschaftliche Aspekte, 688-707. s.1.: Springer. 
Gegner, Martin und O. Schwedes. 2014. Der Verkehr des Leviathan. Zur historischen Genese des städtischen Verkehrs im Rahmen der Daseinsvorsorge. In: O. Schwedes, Hrsg. Öffentliche Mobilität. Perspektiven für eine nachhaltige Verkehrsentwicklung. 2., aktualisierte und erw. Aufl., 47-68. Wiesbaden: Springer VS.

Gerike, Regine. 2006. Wie kann das Leitbild nachhaltiger Verkehrsentwicklung konkretisiert werden? Ableitung grundlegender Aufgabenbereiche. Dresden.

Gertz, Carsten. 2013. Raumwiderstände zwischen Freiheit und Zwang. In: O. Schwedes, Hrsg. Räumliche Mobilität in der zweiten Moderne. Freiheit und Zwang bei Standortwahl und Verkehrsverhalten, 39-58. Münster: LIT.

Giesecke, Raphael, T. Surakka und M. Hakonen. 2016. Conceptualising Mobility as a Service. A User Centric View on Key Issues of Mobility Services. In: Eleventh International Conference on Ecological Vehicles and Renewable Energies (EVER).

Giesel, Flemming und C. Nobis. 2016. The Impact of Carsharing on Car Ownership in German Cities. Transportation Research Procedia 19: 215-224. https://doi. org/10.1016/j.trpro.2016.12.082.

Gouthier, Matthias H.J. und C. Nennstiel. 2018. Neue Mobilitätskonzepte - Eine konzeptionelle Analyse. In: M. Bruhn und K. Hadwich, Hrsg. Service Business Development, 568-589. Wiesbaden: Springer Gabler.

Habermas, Jürgen. 1991. Strukturwandel der Öffentlichkeit. Untersuchungen zu einer Kategorie der bürgerlichen Gesellschaft; mit einem Vorwort zur Neuauflage. 2. Aufl. Frankfurt am Main: Suhrkamp.

Harman, Reg, W. Veeneman und P. Harman. 2012. Innovation in Public Transport. In: F. W. Geels, R. Kemp, G. Dudley und G. Lyons, Hrsg. Automobility in transition? A sociotechnical analysis of sustainable transport, 286-307. New York: Routledge.

Hasse, Felix, M. Jahn, J. N. Ries, M. Wilkens, A. Barthelmess, D. Heinrichs und M. Goletz. 2017. Digital mobil in Deutschlands Städten.

Hensher, David A. und C. Mulley. 2020. Special issue on developments in Mobility as a Service (MaaS) and intelligent mobility. Transportation Research Part A: Policy and Practice 131: 1-4. https://doi.org/10.1016/j.tra.2019.09.039.

Hoffjann, Olaf und H.-J. Arlt. 2015. Öffentlichkeit als Funktionssystem. In: O. Hoffjann und H.-J. Arlt, Hrsg. Die nächste Öffentlichkeit, 7-36. Wiesbaden: Springer Fachmedien Wiesbaden.

Institut für Mobilitätsforschung, Hrsg. 2006. Öffentlicher Personennahverkehr. Herausforderungen und Chancen. Berlin, Heidelberg: Springer Berlin Heidelberg.

Jarren, Otfried und P. Donges. 2011. Politische Kommunikation in der Mediengesellschaft. Wiesbaden: VS Verlag für Sozialwissenschaften.

Jittrapirom, Peraphan, V. Caiati, A.-M. Feneri, S. Ebrahimigharehbaghi, M. J. A. González und J. Narayan. 2017. Mobility as a Service: A Critical Review of Definitions, Assessments of Schemes, and Key Challenges. Urban Planning 2(2): 13-25. https://doi. org/10.17645/up.v2i2.931.

Kagermeier, Andreas. 2011. Verkehrsgeographie. In: H. Gebhardt, R. Glaser, U. Radtke und P. Reuber, Hrsg. Geographie. Physische Geographie und Humangeographie, 735749. Heidelberg: Spektrum Akad. Verl.

Kamargianni, M. und M. Matyas. 2017. The Business Ecosystem of Mobility as a Service. In: $96^{\text {th }}$ Transportation Research Board (TRB) Annual Meeting. 
Karl, Astrid. 2008. Öffentlicher Verkehr im Gewährleistungsstaat. Der ÖPNV zwischen Regulierung und Wettbewerb. Berlin: edition sigma.

Karl, Astrid. 2014. Strukturelle Reformblockaden im Öffentlichen Verkehr. Zu den Herausforderungen von Organisation und Rechtsrahmen. In: O. Schwedes, Hrsg. Öffentliche Mobilität. Perspektiven für eine nachhaltige Verkehrsentwicklung. 2., aktualisierte und erw. Aufl., 71-96. Wiesbaden: Springer VS.

Karlsson, I.C.M., D. Mukhtar-Landgren, G. Smith, T. Koglin, A. Kronsell, E. Lund, S. Sarasini und J. Sochor. 2020. Development and implementation of Mobility-as-aService - A qualitative study of barriers and enabling factors. Transportation Research Part A: Policy and Practice 131: 283-295. https://doi.org/10.1016/j.tra.2019.09.028.

König, Rainer. 2007. Qualität des ÖPNV. https://www.forschungsinformationssystem.de/ servlet/is/237092/https://www.forschungsinformationssystem.de/servlet/is/237092/. Zugegriffen: 4. April 2020.

Kutter, Eckhard. 2016. Siedlungsstruktur und Verkehr: Zum Verständnis von Sachzwängen und individueller Verkehrserreichbarkeit in Stadtregionen. In: O. Schwedes, W. Canzler und A. Knie, Hrsg. Handbuch Verkehrspolitik. 2. Aufl. 2016, 211-236. Wiesbaden: Springer Fachmedien Wiesbaden.

Lanzendorf, Martin und J. Hebsaker. 2017. Mobilität 2.0 - Eine Systematisierung und sozial-räumliche Charakterisierung neuer Mobilitätsdienstleistungen. In: M. Wilde, M. Gather, C. Neiberger und J. Scheiner, Hrsg. Verkehr und Mobilität zwischen Alltagspraxis und Planungstheorie. Ökologische und soziale Perspektiven, 135-151. Wiesbaden: Springer VS.

Lyons, Glenn. 2012. Visions for the Future and the Need for a Social Science Perspective in Transport Studies. In: F. W. Geels, R. Kemp, G. Dudley und G. Lyons, Hrsg. Automobility in transition? A socio-technical analysis of sustainable transport, 29-48. New York: Routledge.

Lyons, Glenn. 2018. Getting smart about urban mobility - Aligning the paradigms of smart and sustainable. Transportation Research Part A: Policy and Practice 115: 4-14. https://doi.org/10.1016/j.tra.2016.12.001.

Matyas, Melinda und M. Kamargianni. 2018. The potential of mobility as a service bundles as a mobility management tool. Transportation 18(1). https://doi.org/10.1007/s11116018-9913-4.

Menzl, Marcus. 2013. Subjektive Raumwiderstände. Die Relevanz lokaler Bindungen bei der Konstitution von räumlichen Alltagsmustern. In: O. Schwedes, Hrsg. Räumliche Mobilität in der zweiten Moderne. Freiheit und Zwang bei Standortwahl und Verkehrsverhalten, 59-76. Münster: LIT.

Meyer, Uli. 2014. Zum Verhältnis von Kollektiv- und Individualverkehr. Oder warum Versuche einer Zusammenführung zweier unterschiedlicher organisationaler Felder scheitern. In: O. Schwedes, Hrsg. Öffentliche Mobilität. Perspektiven für eine nachhaltige Verkehrsentwicklung. 2., aktualisierte und erw. Aufl., 169-187. Wiesbaden: Springer VS.

Nuhn, Helmut und M. Hesse. 2006. Verkehrsgeographie. UTB. 2687. Paderborn: Schöningh. 
Pavone, Marco. 2015. Autonomous Mobility-on-Demand Systems for Future Urban Mobility. In: M. Maurer, J. C. Gerdes, B. Lenz und H. Winner, Hrsg. Autonomes Fahren. Technische, rechtliche und gesellschaftliche Aspekte, 399-416. s.1.: Springer.

Rammler, Stephan und T. Sauter-Servaes. 2013. Innovative Mobilitätsdienstleistungen. Arbeitspapier. 274.

Rammler, Stephan. 2014. Im Omnibus in die Moderne. Öffentliche Verkehrssysteme im Spiegel gesellschaftlicher Modernisierung. In: O. Schwedes, Hrsg. Öffentliche Mobilität. Perspektiven für eine nachhaltige Verkehrsentwicklung. 2., aktualisierte und erw. Aufl., 25-46. Wiesbaden: Springer VS.

Rees, Dagmar. 2018. Digitalisierung in Mobilität und Verkehr. Schiene und öffentlicher Verkehr. Bingen: PMC Media House GmbH.

Rölle, Daniel. 2005. Einflussfaktoren geänderten Mobilitätsverhaltens auf Arbeits- und Freizeitwegen. Zugl.: Stuttgart, Univ., Diss., 2005. Empirische und methodologische Beiträge zur Sozialwissenschaft. 22. Frankfurt am Main: Lang.

Rürup, Bert und S. Jung. 2017. Digitalisierung: Chancen auf neues Wachstum. In: A. Hildebrandt und W. Landhäußer, Hrsg. CSR und Digitalisierung. Der digitale Wandel als Chance und Herausforderung für Wirtschaft und Gesellschaft, 3-21. Berlin, Heidelberg: Springer Berlin Heidelberg.

Schade, Jens und B. Schlag. 2007. Psychologie des Mobilitätsverhaltens. Aus Politik und Zeitgeschichte (29-30/2007): 27-32.

Schlag, Bernhard und L. Rößger. 2019. Carsharing - Motive und Intentionen. Report Psychologie 2 (2019) (45).

Schmoll, Carsten, J. Tiemann und C. Welzel. 2014. Digitale Mobilität - Dynamik im öffentlichen Raum. Berlin.

Schneider, Uta. 2018. Urbane Mobilität im Umbruch. Normen, Leitbilder und familiäre Aushandlungsprozesse zu Autos und Elektroautos. Studien zur Mobilitäts- und Verkehrsforschung. Wiesbaden: Springer Fachmedien Wiesbaden.

Schnieder, Lars und L. Gebhardt. 2016. Nutzerorientierter Entwurf innovativer Mobilitätskonzepte für urbane Räume. Magdeburg. 14. Fachtagung EKA 2016 - Entwurf komplexer Automatisierungssysteme.

Schwedes, Oliver und A. Rammert. 2020. Was ist Integrierte Verkehrsplanung? Hintergründe und Perspektiven am Menschen orientierter Planung. Berlin. IVP-Discussion Paper. 2020(2).

Schwedes, Oliver, S. Daubitz, A. Rammert, B. Sternkopf und M. Hoor. 2018. Kleiner Begriffskanon der Mobilitätsforschung. 2. Auflage. IVP-Discussion Paper. 2018(1).

Schwedes, Oliver. 2014a. Einleitung: Scheitern als Chance! In: O. Schwedes, Hrsg. Öffentliche Mobilität. Perspektiven für eine nachhaltige Verkehrsentwicklung. 2., aktualisierte und erw. Aufl., 13-22. Wiesbaden: Springer VS.

Schwedes, Oliver. 2014b. Fazit: Vom Öffentlichen Verkehr zu Öffentlichen Mobilität. In: O. Schwedes, Hrsg. Öffentliche Mobilität. Perspektiven für eine nachhaltige Verkehrsentwicklung. 2., aktualisierte und erw. Aufl., 241-251. Wiesbaden: Springer VS.

Stadtraum. 2019. BVG Mobilitäts-Hub. https://www.stadtraum.com/stadtraum/ aktuelles1906.html. Zugegriffen: 24. Juni 2020. 
Trapeze Switzerland GmbH. 2020. New Mobility: Bedarfsorientierte öffentliche Mobilität. https://www.trapezegroup.de/de/bedarfsverkehr/details/bedarfsorientierte-oeffentlichemobilitaet/. Zugegriffen: 2. April 2020.

Verband Deutscher Verkehrsunternehmen (VDV). 2019. Smart Mobility - Neue Chancen für die öffentliche Mobilität. https://www.vdv.de/neue-chancen-fuer-die-oeffentlichemobilitaet.aspx. Zugegriffen: 2. April 2020.

Zhou, Fan, Z. Zheng, J. Whitehead, R. K. Perrons, S. Washington und L. Page. 2020. Examining the impact of car-sharing on private vehicle ownership. Transportation Research Part A: Policy and Practice 138: 322-341. https://doi.org/10.1016/j. tra.2020.06.003.

Open Access Dieses Kapitel wird unter der Creative Commons Namensnennung 4.0 International Lizenz (http://creativecommons.org/licenses/by/4.0/deed.de) veröffentlicht, welche die Nutzung, Vervielfältigung, Bearbeitung, Verbreitung und Wiedergabe in jeglichem Medium und Format erlaubt, sofern Sie den/die ursprünglichen Autor(en) und die Quelle ordnungsgemäß nennen, einen Link zur Creative Commons Lizenz beifügen und angeben, ob Änderungen vorgenommen wurden.

Die in diesem Kapitel enthaltenen Bilder und sonstiges Drittmaterial unterliegen ebenfalls der genannten Creative Commons Lizenz, sofern sich aus der Abbildungslegende nichts anderes ergibt. Sofern das betreffende Material nicht unter der genannten Creative Commons Lizenz steht und die betreffende Handlung nicht nach gesetzlichen Vorschriften erlaubt ist, ist für die oben aufgeführten Weiterverwendungen des Materials die Einwilligung des jeweiligen Rechteinhabers einzuholen.

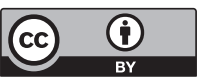

